\title{
UV line intensity and flow velocity distributions in two coronal mass ejections as deduced by UVCS-SOHO observations
}

\author{
R. Ventura ${ }^{1}$, D. Spadaro ${ }^{1}$, M. Uzzo $^{2}$, and R. Suleiman ${ }^{2}$ \\ 1 Osservatorio Astrofisico, Città Universitaria, Via S. Sofia 78, 95125 Catania, Italy \\ 2 Harvard-Smithsonian Center for Astrophysics, Cambridge, MA 02138, USA \\ A\&A 383, 1032-1048 (2002), DOI: 10.1051/0004-6361:20011802
}

This article has been misprinted. Following this page the article will be printed again with its original page numbers. 


\title{
UV line intensity and flow velocity distributions in two coronal mass ejections as deduced by UVCS-SOHO observations
}

\author{
R. Ventura ${ }^{1}$, D. Spadaro ${ }^{1}$, M. Uzzo ${ }^{2}$, and R. Suleiman ${ }^{2}$ \\ 1 Osservatorio Astrofisico, Città Universitaria, Via S. Sofia 78, 95125 Catania, Italy \\ 2 Harvard-Smithsonian Center for Astrophysics, Cambridge, MA 02138, USA
}

Received 10 July 2001 / Accepted 14 December 2001

\begin{abstract}
The Ultraviolet Coronagraph Spectrometer (UVCS) instrument onboard the SOHO satellite observed two coronal mass ejections on November 2 and 3,2000, related to the eruptions of a large filament structure in an active region close to the West limb of the Sun, and of a prominence near the South Pole, respectively. Intensity and profile of the O VI resonance doublet lines at 1032 and $1037 \AA$ and of Ly $\beta$ (1026 $\AA)$ line, together with the intensity of some other minor ions, were observed using the O VI channel of UVCS. We analysed these spectroscopic observations in order to get information about the distributions of ionic densities and flow velocities in the solar coronal plasma ejected during these transient events. Emission in ions ranging from C II to O VI indicates a temperature range between $10^{4.5}$ and $10^{5.5} \mathrm{~K}$. The morphology of the bright emission regions suggests the development of several strands of plasma irregularly distributed inside the CME structures, whose temporal evolution is significantly different from each other. The velocities determined for each bright element also give a complex picture of the plasma kinematics characterizing these coronal mass ejections.
\end{abstract}

Key words. Sun: corona - Sun: UV radiation

\section{Introduction}

Since their first detection in the early 1970's, coronal mass ejections (CMEs) have been extensively observed and studied (e.g., Hundhausen et al. 1988, 1997; Wagner 1984). Nevertheless many questions are still unresolved: their precursors, origins and onset, for instance, are not yet well established. They often appear associated with prominence eruptions or with solar flares and sometimes also with quiescent filaments, but there is as well evidence of a correlation with non-equilibrium configurations of larger scale magnetic fields. On the other hand, the key role played by the magnetic field in triggering and structuring the ejecta and handling their evolution is not fully understood, as well as the effects of CMEs on the solar cycle and global magnetic field restructuring (e.g., Low 1996).

The present models of CMEs are not well constrained by observations. In most of them (e.g. Sturrock 1989; Moore \& Roumeliotis 1992; Low 1994; Chen 1996) magnetic helicity, associated with both untwisted and twisted magnetic fields, is central to explain morphology, dynamics and energetics of some observed CMEs (e.g., Antonucci et al. 1997). The helical structure, conversely, is not

Send offprint requests to: R. Ventura,

e-mail: rventura@alpha4.ct.astro.it required in other models, based on reconnection in magnetic loops (Antiochos et al. 1999), which attempt to describe the eruption as driven uniquely by magnetic free energy stored in a closed, sheared arcade. Since the coronal plasma is confined and flows along the magnetic field lines, detailed determinations of the velocity distributions in the observed ejecta might help to discriminate between these two possibilities.

Most of the past CME observations have been performed using space- and ground-based white-light coronagraphs with a limited field of view (from 1.2 to $10 R_{\odot}$ ), preventing us from collecting crucial information on their precursors, onset and earliest stage of evolution. On the other hand, a multiwavelength approach is strongly required in order to follow the complete development of a $\mathrm{CME}$, which involves eruption of both hot $\left(10^{6} \mathrm{~K}\right)$ coronal plasma and cool $\left(10^{4} \mathrm{~K}\right)$ prominence material, with a strong precursory activity at chromospheric and low corona levels. Hence, coordinated observations can provide new insights on the physical and dynamical conditions of the ejected material, which in turn can put constraints on the theoretical models.

In the past decade, the Soft X-Ray Telescope (SXT) aboard the Yohkoh satellite, the radio imaging techniques (e.g., Maia et al. 1999, 2000) and even more effectively the coronal instruments aboard the $\mathrm{SOHO}$ mission 
(Domingo et al. 1996), have provided this possibility. The SXT identified X-ray depletions as probable signatures of the CME onset, and detected soft X-ray emission in the range $2-6 \times 10^{6} \mathrm{~K}$ during coronal eruptions. Radio observations opened the possibility to forecast CME appearence in the solar corona (e.g., Durasova et al. 1999) through the detection of nonstationary radio events which seem to occur at the earliest stages of the eruption. White-light observations of CMEs by LASCO (Large Angle Spectrometric COronagraph - Brueckner et al. 1995) on board SOHO provide information on the electron density and velocity projected on the plane of the sky over a large range of heliocentric distance, out to $30 R_{\odot}$. UV imaging and spectroscopy performed by EIT (EUV Imaging Telescope - Delaboudiniere et al. 1995) and UVCS (UltraViolet Coronagraph Spectrometer - Kohl et al. 1995) give information on the chromospheric and coronal emissions over a large range of temperature, allowing detailed diagnostics of velocity fields, temperatures, densities, ionization state and elemental abundances. Moreover, the different perspectives provided by images in EUV spectral lines and in white-light can be used to derive information on the $3 \mathrm{D}$ velocity structure characterizing the CME, which in turn can constrain its magnetic topology.

In this paper we present UVCS observations of two CMEs which occurred in two consecutive days (November 2 and 3, 2000) during the MEDOC (MultiExperiment Data Operations Center for SOHO) campaign \# 6, held in October - November 2000. The ejection of November 2 was detected at the West limb of the Sun, near the equator, and allowed to study the region following the front of the event. During November 3, a mass ejection occurring near the South Pole of the Sun has been followed since its beginning until the end, while the front of the event crossed the UVCS instantaneous field of view.

The paper is organized as follows: the observational details are reported in Sect. 2, the results of our analysis, concerning the distributions of some EUV line intensities and of flow velocities in the ejected plasma, are presented and discussed in Sect. 3, while in Sect. 4 we draw some conclusions.

\section{Observations and data analysis}

The UltraViolet Coronagraph Spectrometer on board $\mathrm{SOHO}$ is able to perform ultraviolet observations of the extended corona in the range of heliocentric distances 1.5-10 $R_{\odot}$. Intensity and profile of strong EUV emission lines, such as the $\mathrm{H} \mathrm{I} \mathrm{Ly} \alpha$ and $\operatorname{Ly} \beta$ and the O VI resonance doublet at $1032 \AA$ and $1037 \AA$, together with some other minor ion lines, can be measured.

During the MEDOC campaign \# 6, coordinated at the Institut d'Astrophisique Spatiale in Orsay - Paris, UVCS, EIT and LASCO detected two strong CMEs. On November 2, 2000 UVCS observations were devoted to a radial scan of the corona above the West limb of the Sun at a PA of $270^{\circ}$ (measured counterclockwise from the North
Table 1. Observing sequence of November 2 CME.

\begin{tabular}{|ccc|}
\hline $\begin{array}{c}\text { Heliocentric } \\
\text { distance } \\
\left(R_{\odot}\right)\end{array}$ & $\operatorname{Exps}^{a}$ & Start time \\
\hline 1.5 & 9 & $18: 45: 21$ \\
1.6 & 9 & $19: 17: 24$ \\
1.7 & 9 & $19: 49: 29$ \\
1.8 & 12 & $20: 21: 44$ \\
2.0 & 12 & $21: 06: 08$ \\
2.2 & 12 & $21: 51: 00$ \\
2.5 & 18 & $22: 35: 23$ \\
3.0 & 18 & $23: 40: 50$ \\
\hline${ }^{a} 200$ s each. & & \\
\hline
\end{tabular}
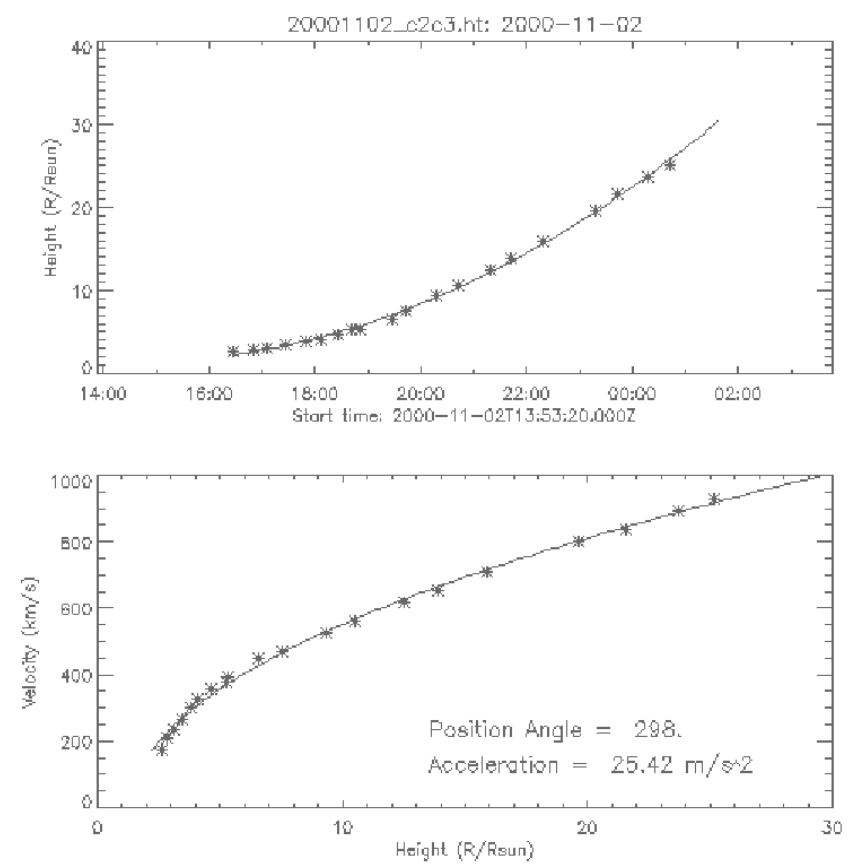

Fig. 1. Height vs. time and velocity vs. height of the CME leading edge, as determined by LASCO C2 and C3 observations (by courtesy of S. Plunckett).

Pole). The equatorial region was scanned from 1.5 to $3 R_{\odot}$ with exposures of $200 \mathrm{~s}$ each. Details of the observation sequence are reported in Table 1 . Intensity and profile of the OVI resonance doublet lines at $1032 \AA$ and $1037 \AA$ and of the $\operatorname{Ly} \beta(1026 \AA)$ line, together with the intensity of some other minor ions (such as the C III $977 \AA$ line), were observed using the O VI channel of UVCS over spatial elements of $14^{\prime \prime} \times 42^{\prime \prime}$, in a slice of the corona $40^{\prime}$ long. The slit width was $50 \mu \mathrm{m}$ in the $1.5-2.0 R_{\odot}$ range of heliocentric distance and $150 \mu \mathrm{m}$ between $2.2 R_{\odot}$ and $3.0 R_{\odot}$. The spectral bin corresponds to $0.0993 \AA$ and $0.199 \AA$, respectively, for the two ranges of heliocentric distance.

UVCS observations started at 18:45 UT, more than two hours later than LASCO first detection of the coronal mass ejection: the event located at a PA of about $315^{\circ}$, 

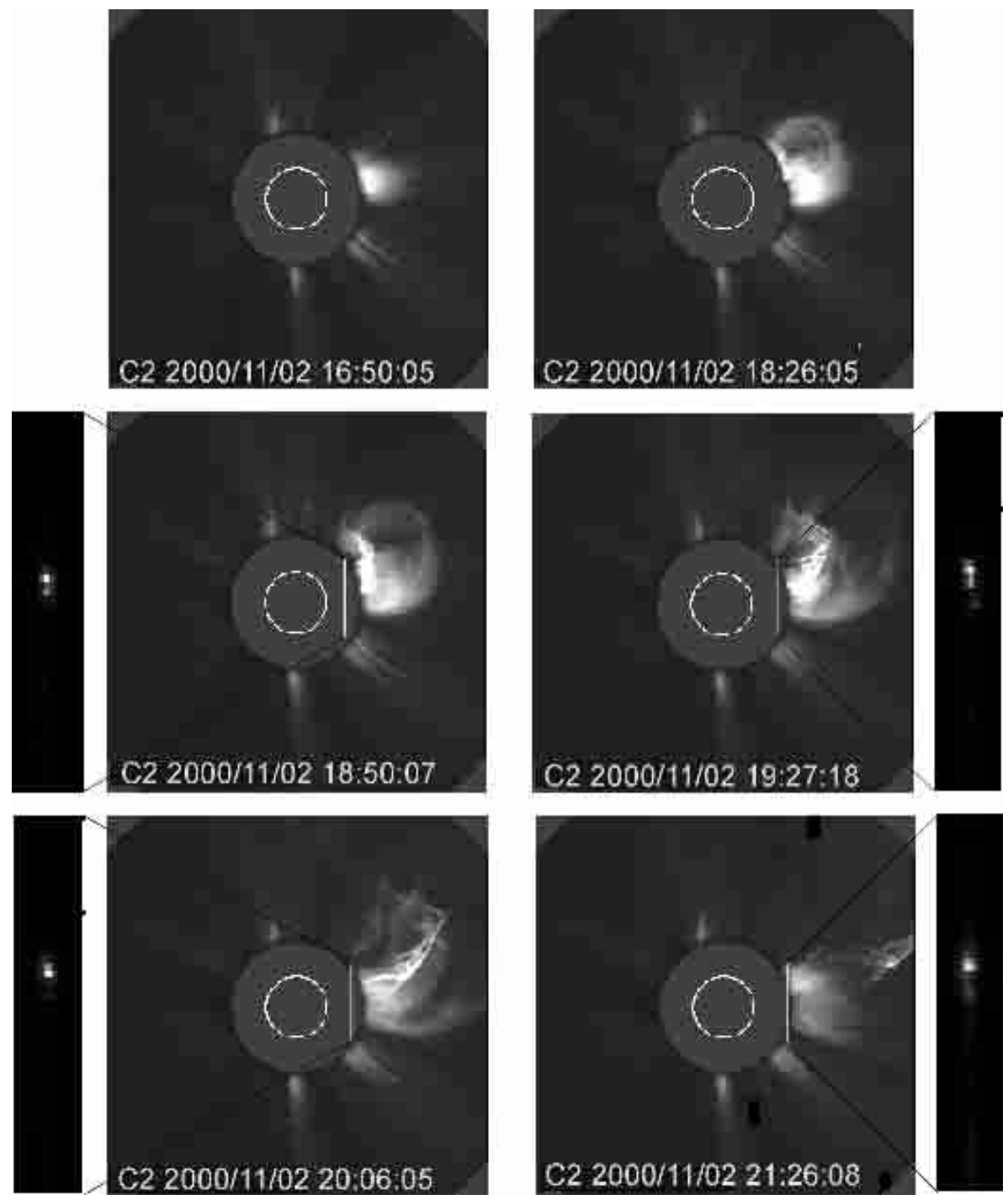

Fig. 2. Sequence of LASCO C2 images taken on November 2, 2000 during the progression of the CME. The instantaneous positions of the UVCS slit at the time of the observation (white segments) are also shown, together with the corresponding O VI $1032 \AA$ slit profiles. The vertical and horizontal axes are the spatial coordinates along the slit and the wavelengths, respectively. Shorter wavelengths are at right and smaller polar angles at the bottom of each panel. The vertical extent is 40 arcmin, the covered wavelength range is $3.76 \AA$. The UVCS slit is centered at a PA equal to $270^{\circ}$, and at $1.5 R_{\odot}(18: 50 \mathrm{UT})$, $1.6 R_{\odot}(19: 27 \mathrm{UT}), 1.7 R_{\odot}(20: 06 \mathrm{UT})$ and $2 R_{\odot}(21: 26 \mathrm{UT})$, respectively.

entered the field of view of LASCO C2 coronagraph $\left(2-6 R_{\odot}\right)$ at 16:26 UT (Plunckett, private communication). As detected by LASCO $\mathrm{C} 2$ and $\mathrm{C} 3\left(3.7-30 R_{\odot}\right)$ coronagraphs, the event started out slowly (Fig. 1) then accelerated in their fields of view: at $2.6 R_{\odot}$ the speed of the leading edge, projected on the plane of the sky, was $170 \mathrm{~km} \mathrm{~s}^{-1}$, then it increased up to $930 \mathrm{~km} \mathrm{~s}^{-1}$ at $25 R_{\odot}$ (Plunckett, private communication). In white-light radiation it appeared first as a bright front with a small angular extension (Fig. 2, left top panel). Throughout the progression of the $\mathrm{CME}$, it expanded and a large loop with a very bright core and a fainter leading edge developed in about two hours (Fig. 2, right top panel and left middle panel). Later on, at larger heliocentric distances, (Fig. 2, right middle panel and left bottom panel) a long, complex outflow appeared to twist and turn towards high latitudes. At 21:26 UT the CME almost faded out (Fig. 2, right bottom panel). The CME bright structures seen in white-light radiation are also detectable in the UVCS longslit OVI $1032 \AA$ profiles as shown in Fig. 2. Moreover the UVCS image of the radial scan obtained in the O VI $1032 \AA$ radiation from 18:45 UT to 00:45 UT (Fig. 3) shows a CME morphology in the ultraviolet very similar to that shown by the LASCO $\mathrm{C} 2$ white-light image: we can identify a bright core surrounded by two well defined structures which elongate at larger height toward higher latitudes, instead of propagating radially. The Ly $\beta$ and C III $977 \AA$ images of the CME show similar morphological characteristics.

EIT He II $304 \AA$ and Fe XV $284 \AA$ images taken the day before, November 1, 2000 (Figs. 4 and 5, left panels), reveal the presence of a multi-structured large active 
Polar Angle $=270 \mathrm{Deg}$
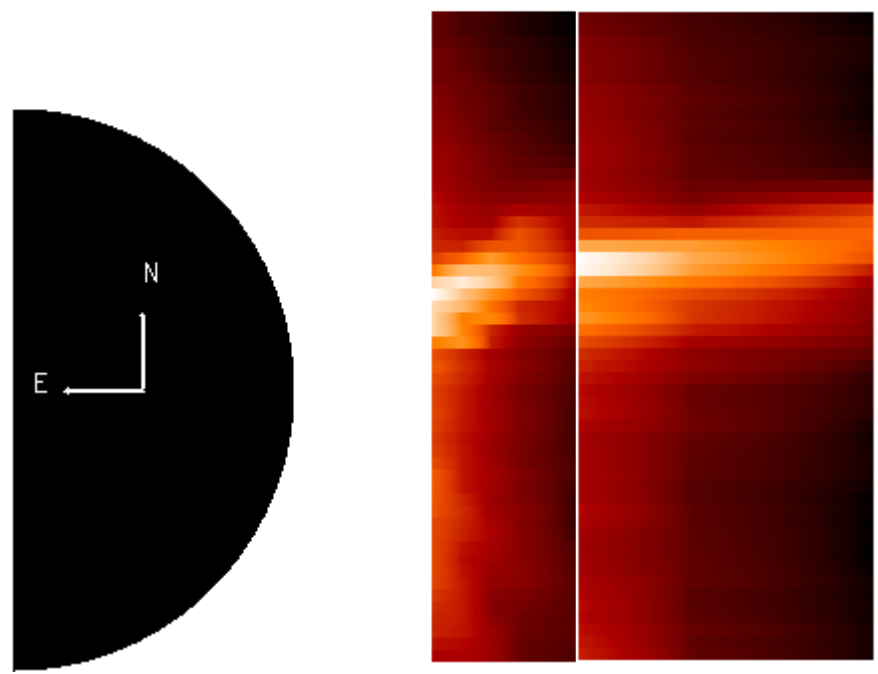

Fig. 3. UVCS O VI $1032 \AA$ image of the CME of November 2, 2000 obtained from the radial scan at the solar equator $\left(1.5-3.0 R_{\odot}\right)$ performed in about six hours of observations. The left panel refers to the continuous sequence in the range 1.5-2.0 $R_{\odot}$ from 18:45 UT until 21:49 UT. Then UVCS changed its instrumental configuration and scanned the equatorial region in the range $2.2-3.0 R_{\odot}$ from 22:00 UT until 00:42 UT (right panel).

region, close to the West limb of the Sun, at a PA of about $315^{\circ}$, where a large dark filament (more evident in the $304 \AA$ band and still detectable in the other two bandpasses of the instrument at $171 \AA$ and $195 \AA$ ) stands out from the diffuse emission of the background. A very bright area, apparently related to the filament, is also present in the EIT Fe IX/X $171 \AA$, Fe XII $195 \AA$ (not shown) and Fe XV $284 \AA$ (Fig. 5, left panel) images. EIT's images taken the day of the event clearly show the ejection of plasma (Figs. 4 and 5, middle panels). A visual inspection of the EIT movies (kindly provided by Delaboudiniere), performed in all the four band-passes of the instrument, points out that a large part of the active region (larger than it appears in the images shown here) is involved in the explosive event. In the EIT $195 \AA$ movie the dark filament, detected the day before, appears to erupt at 15:36 UT. A new active arch-like region, probably caused by the magnetic field restructuring after the CME occurrence (Fig. 5, middle panel), forms and evolves in about two hours after the eruption, as it is evident in all the higher temperature band-pass movies and images, while it is not detected in the He II $304 \AA$ image (Fig. 4, middle panel) which forms at significantly lower temperature. A void in the coronal emission in the region where the CME developed is detectable in the EIT images taken the day after the event (Figs. 4 and 5, right panels). The UVCS slit position at the time of the CME occurrence together with the OVI 1037 and $1032 \AA$, and the Ly $\beta$ long-slit profiles are also reported in Figs. 4 and 5. It appears that the jet material clearly seen in the $304 \AA$ image and more faintly in the $284 \AA$ one extends continuously into the UVCS
Table 2. Observing sequence of November 3 CME.

\begin{tabular}{|ccc|}
\hline $\begin{array}{c}\text { Heliocentric } \\
\text { distance } \\
\left(R_{\odot}\right)\end{array}$ & $\operatorname{Exps}^{a}$ & Start time \\
\hline 1.50 & 15 & $18: 17: 44$ \\
1.55 & 18 & $19: 10: 47$ \\
1.60 & 18 & $20: 14: 19$ \\
1.50 & 15 & $21: 17: 46$ \\
1.55 & 18 & $22: 10: 44$ \\
1.60 & 18 & $23: 14: 11$ \\
\hline${ }^{a}$ 200 s each. & & \\
\end{tabular}

instantaneous field of view, producing the bright features detected in the long-slit profiles.

The second coronal mass ejection was detected on November 3, 2000. The CME, started at around 19:00 UT, as deduced by EIT and LASCO observations (Delaboudiniere and Plunckett, respectively, private communications), reached the UVCS instantaneous field of view at $1.55 R_{\odot}$ at 19:56 UT, 1 hour and 40 min later than the beginning of the UVCS observations (started at 18:17 UT), which were devoted to monitor the evolution of the coronal environment above a prominence located near the South Pole, at a PA of about $200^{\circ}$. A radial scan from 1.5 to $1.6 R_{\odot}$, with exposures of $200 \mathrm{~s}$ each and a duration of about 3 hours was repeated twice. Table 2 reports the details of the observing sequence.

The instrumental configuration and the lines observed were the same as for the previous day. The slit was centered at a PA equal to $200^{\circ}$ and its width was taken constant at $50 \mu \mathrm{m}$ at any position. UVCS followed all the evolution of the prominence eruption until its disappearence from the instrument field of view at about 22:03 UT; UVCS observations ended at 00:16 UT.

Unfortunately LASCO C2 and C3 coronagraphs were not able to get clear images of the event, which was probably part of a tenous halo detected in white-light over the South Pole. However, it was possible to determine a leading edge speed (projected on the plane of the sky) of about $290 \mathrm{~km} \mathrm{~s}^{-1}$, with a coarse accuracy of about $\pm 50 \mathrm{~km} \mathrm{~s}^{-1}$, due to the large scatter of the LASCO measurements (Plunckett, private communication). The EIT He II images of November 2 and November 3 (Fig. 6) clearly show the 24 -hours evolution of the prominence located at about $200^{\circ}$. The instantaneous position of the UVCS slit at the time of the observations (middle panel), together with the OVI $1032 \AA$ and $1037 \AA$ long-lit profiles (bottom panel), are also reported in Fig. 6. The detailed evolution of the prominence during its eruption is clearly visible in the sequence of EIT $195 \AA$ images shown in Fig. 7 (kindly provided by Delaboudiniere), obtained as differences between successive images (running difference images), in order to increase the sensitivity in detecting motions. The sequence starts at 19:13 UT, just after the eruption and ends at 21:12 UT. The eruption seems 


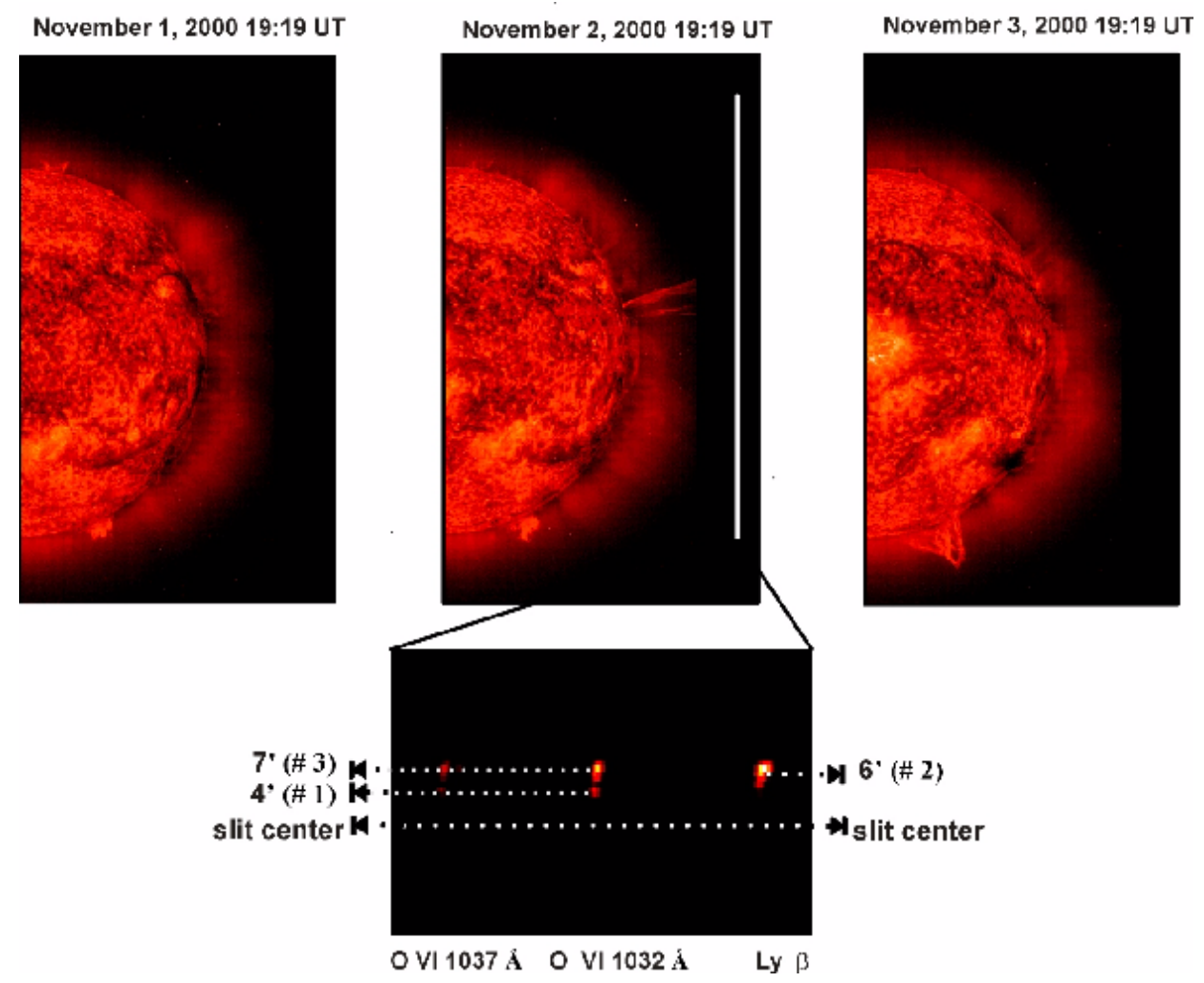

Fig. 4. Sequence of EIT He II $304 \AA$ images of the Sun taken the day before (November 1, 2000 - left panel) the occurrence of the CME, the day of the event (November 2, 2000 - middle panel) and the day after (November 3, 2000 - right panel). The UVCS slit position at the time of the 2 November, 2000 EIT observation (19:19 UT) is also reported together with the OVI and Ly $\beta$ long-slit profiles. The vertical and horizontal axes are the spatial coordinates along the slit and the wavelengths, respectively. Shorter wavelengths are at right and smaller polar angles at the bottom of each panel. The vertical extent is 40 arcmin; the covered wavelength range is $16 \AA$. The slit center, defined as the slit region of minimum heliocentric distance is located at a PA equal to $270^{\circ}$. It is shown together with three bright spots identified and numbered progressively on the basis of their distance from it (in arcmin). The grey scale of the He II $304 \AA$ has been saturated in order to better distinguish the structures involved in the eruption.

to affect more the prominence in the transverse direction than in the radial one. The footpoints of the prominence appear to move away in opposite direction more quickly than its front with a roughly estimated mean velocity of about $5 \mathrm{~km} \mathrm{~s}^{-1}$ in the radial direction and $50 \mathrm{~km} \mathrm{~s}^{-1}$ in the transverse one.

The UVCS Data Analysis and processing Software (DAS, originally developed by C. Benna, A. van Ballegooijen, J. Raymond and S. Giordano) was used to remove image distorsion, to flat-field the detectors, to perform the wavelength and radiometric calibration of the data and to extract the spectra (Gardner et al. 1996; Kohl et al. 1997a, 1997b; Romoli et al. 2001). Stray light subtraction and instrumental broadening correction was also performed using Panasyuk's package (Panasyuk et al. 2001). Profiles of the O VI resonance doublet and H I Ly $\beta$ lines were curve-fitted to single Gaussians in order to determine intensity values and peak intensity wavelengths. Doppler shifts of the lines have then been computed in order to derive line-of-sight velocities. The spectral bin adopted for the C III line $977 \AA$ was insufficient to allow us to get a profile, so that a Gaussian fitting was not applicable. The C III line intensities were then computed by adding the contributions at each wavelength in the range covered by the line. The results obtained for the two events are presented in the next section.

\section{Results}

\subsection{The CME of November 2}

\subsubsection{Spectra and line intensities}

Figure 8 shows a sequence of spectra extracted from the data of November 2 and taken in the wavelength regions $1022-1045 \AA$, and $970-978 \AA$. The adopted spectral bins correspond to $0.0993 \AA$ and $0.298 \AA$, respectively. The most prominent feature in the spectra taken at $1.5 R_{\odot}$ (18:45 UT) is the chromospheric H I Ly $\beta$ line, which is even more intense than the coronal O VI doublet lines (Fig. 8a). Also the two chromospheric lines of $\mathrm{C}$ II at $1037.02 \AA$ and $1036.34 \AA$ are evident in the blue wing of the O VI 1037.61 $\AA$ line. The spectral region $970-978 \AA$ is dominated by the very intense chromospheric C III $977 \AA$ line, while a faint $\mathrm{H} \mathrm{I} \mathrm{Ly} \gamma 972 \AA$ line is detectable just above the noise level (Fig. 8b). The appearence of spectral lines typically formed at chromospheric temperatures 


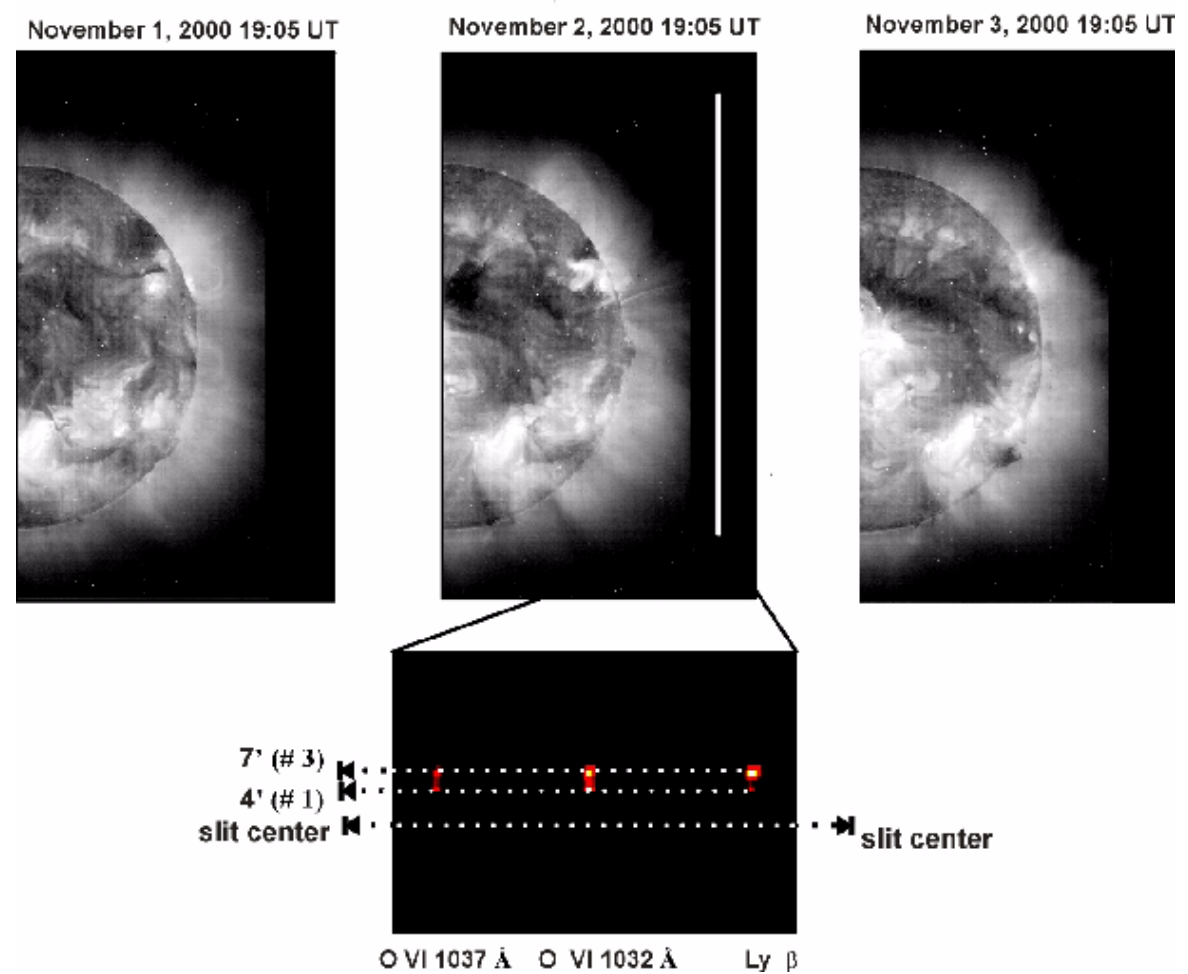

Fig. 5. As in Fig. 4 but for the Fe X V $284 \AA$ band pass.
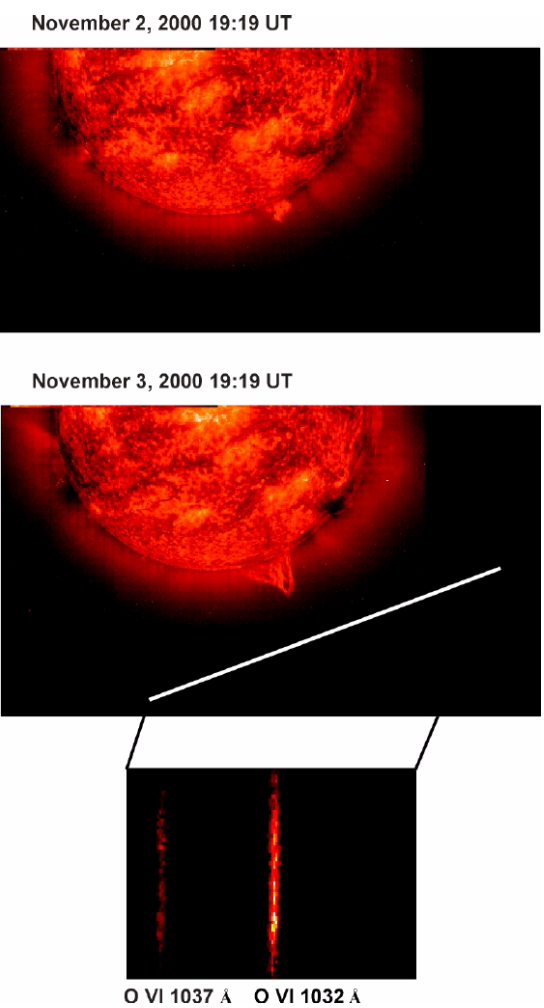

Fig. 6. EIT He II $304 \AA$ images of the solar Southern hemisphere, taken at the same UT (19:19) on November 2, 2000 (top panel) and November 3, 2000 (bottom panel), showing the evolution of the prominence located at a PA of about $200^{\circ}$. The UVCS slit position at the time of the observation (white segment) together with the O VI $1037 \AA$ and $1032 \AA$ slit profiles are also reported. The vertical and spatial scales as well as their ranges are the same as in Fig. 4. The UVCS slit is centered at a PA equal to $200^{\circ}$ and at $1.55 R_{\odot}$. is an evident signature of the presence, at coronal levels, of cooler chromospheric material ejected at large heliocentric distances during the CME. At $1.7 R_{\odot}(20: 06 \mathrm{UT})$, the peak intensities of the Ly $\beta$ and O VI doublet lines are more than one order of magnitude and a factor of three fainter than at $1.5 R_{\odot}$, respectively, while the $\mathrm{C}$ II lines are no longer detected, probably due to the ionization of the chromospheric material (Fig. 8c). In the other panel (Fig. 8d), the C III and the Ly $\gamma$ lines are still visible, although the peak intensity of the former line is more than a factor of seven lower, while the latter one is nearly lost in the noise.

The spectra taken at $2.5 R_{\odot}(22: 46 \mathrm{UT})$ refer to a little bit different spectral regions and were taken with a coarser spectral binning. The first panel (Fig. 8e) covers the wavelength region $997-1046 \AA$ with a spectral binning corresponding to $0.199 \AA$, the second one (Fig. 8f) the spectral region $970-979 \AA$ with a spectral binning corresponding to $0.397 \AA$. The most significant features of the spectral sequence are a decrease of the peak intensity of the $\mathrm{O}$ VI lines and the loss in the noise of the $\mathrm{Ly} \beta, \mathrm{Ly} \gamma$ and C III lines.

A detailed analysis of the intensity distribution of the OVI $1032 \AA, 1037 \AA, \operatorname{Ly} \beta$ and C III lines was then performed, determining the line intensities according to the procedure described at the end of Sect. 2. Since the slit did not appear uniformely bright in the emitted radiation, we identified along the slit and at any heliocentric distance spot-like brightenings, which are parts of CME's global structure (see Figs. 4 and 5 for their location inside the slit and numbering) and continuosly evolve on a wide range of time-scales. It is worth to notice that the 

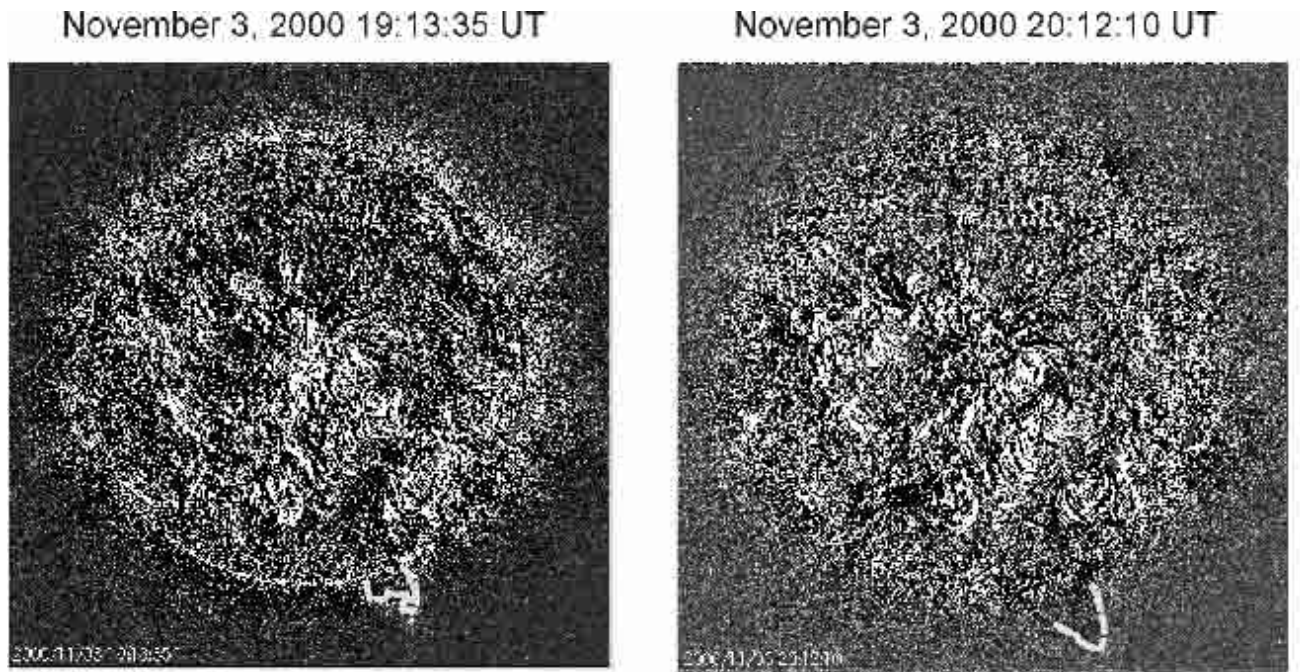

\section{November 3, 2000 20:39:39 UT}

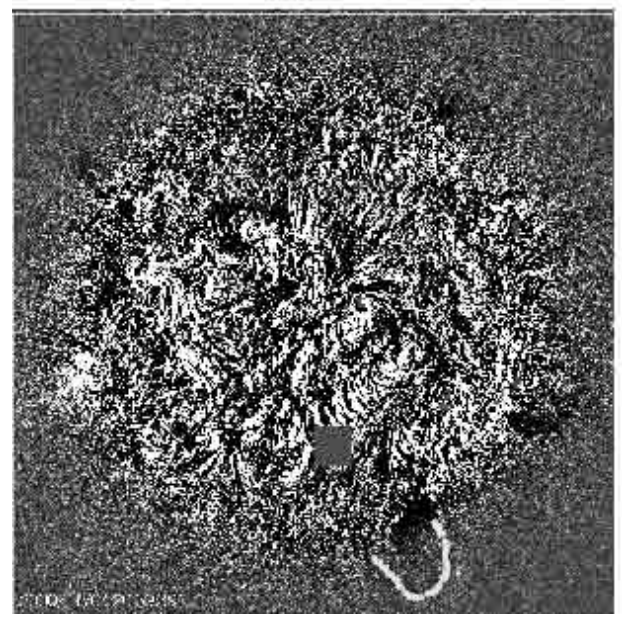

November 3, $200021: 12: 16$ UT

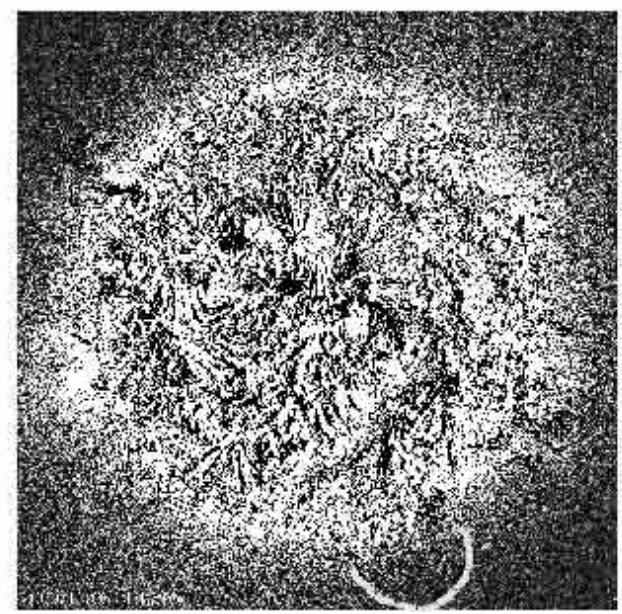

Fig. 7. Sequence of EIT $195 \AA$ A runnig difference images showing the detailed evolution of the prominence eruption of November 3 , 2000. The prominence is enclosed by the white dashed line.

number of bright features, their extension and position along the slit are not the same for all the lines considered. We then computed, for each exposure at any heliocentric distance, the intensity values spatially averaging over each identified spot. We also computed, as a reference level for the quiet corona emission, the intensities of the lines in a less bright section of the slit, far away from the bulk of the CME structure, then reasonably pertaining to the unperturbed quiet corona. In the case of the $\mathrm{O}$ VI lines the values so obtained in the range of heliocentric distance $1.6-1.8 R_{\odot}$ are consistent with those derived from the mini-synoptic observations of November 2, performed before the CME eruption (from $00 \mathrm{UT}$ to 04 UT) at the same solar latitude and at the two heliocentric distances $1.6 R_{\odot}$ and $1.75 R_{\odot}$. Global results are reported in Figs. 9 and 10. The O VI $1032 \AA$ intensity distribution along the slit at any heliocentric distance (Fig. 9) exhibits a rapidly evolving complex morphology of the CME, with quite a few bright knots appearing, intensifying, or weakening and even extinguishing on the short time interval between two consecutive exposures. The intensity values in the various identified bright spots are at least one order of magnitude higher than in the quiet corona. The $\mathrm{Ly} \beta$ intensity distribution (Fig. 10) shows similar characteristics, with an even more complex morphology. For istance at $1.7 R_{\odot}$ at least five different bright substructures are evident, whose intensities continuously change, even relatively to each other. Above $1.8 R_{\odot}$ the line becomes undetectable. The C III intensity distribution is conversely very simple, with only a single bright knot which becomes fainter and fainter for increasing height. At $2.0 R_{\odot}$ its intensity drops with time by more than one order of magnitude. The $\mathrm{C}$ III line disappears above $2.0 R_{\odot}$.

\subsubsection{Velocity structure}

The Gaussian line fitting of the O VI $1032 \AA$ and Ly $\beta$ line profiles also allowed to determine the Doppler shift of the lines with respect to their rest wavelength, due to the motion of the emitting plasma along the line of sight. 
November 2

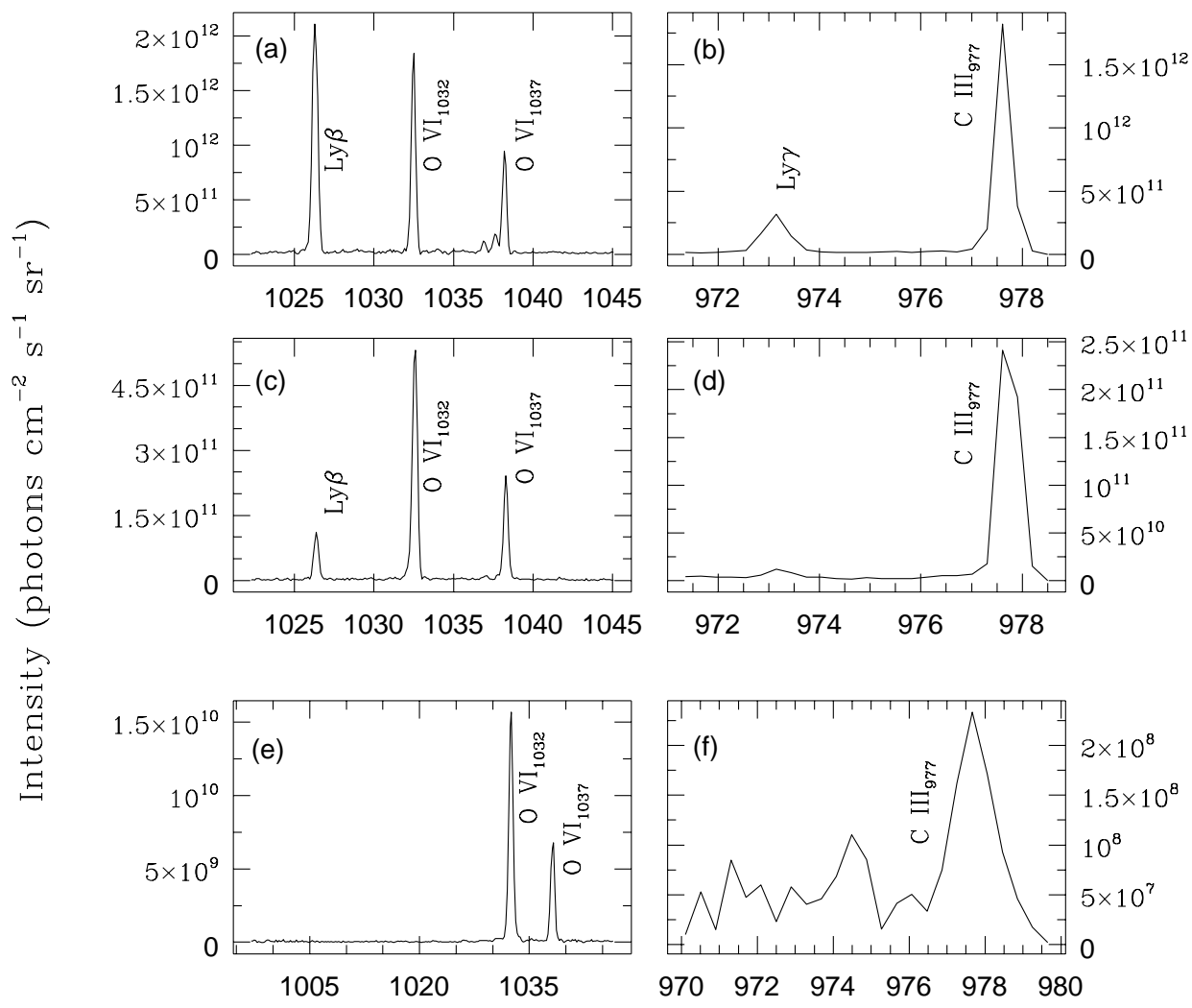

$\lambda(\AA)$

Fig. 8. Sequence of spectra extracted from the UVCS data of November 2, 2000 in the wavelength regions $1022-1045 \AA$ and 970-978 $\AA$ (range of heliocentric distance: 1.5-2.0 $R_{\odot}$ ), and 997-1046 $\AA$ and 970-979 $\AA$ (range of heliocentric distance: $\left.2.2-3.0 R_{\odot}\right)$. The panels refer to the following heliocentric distances and initial exposure times, respectively: a,b) $1.5 R_{\odot}$, 18:45 UT, c,d) $1.7 R_{\odot}, 20: 06$ UT and e,f) $2.5 R_{\odot}, 22: 46$ UT. Note the changes in the vertical scale among different panels. All the spectra have been obtained integrating along the slit over the spatial extent ( $\left.42^{\prime \prime}\right)$ of the very bright spot \# 3 (see Fig. 4 ), located at 7 arcmin (towards larger polar angles) from the slit center.

As for the intensities, we computed this quantity for each bright feature singled out in the CME structure.

The question on correctly interpreting the Doppler shift of a line in terms of velocity along the line of sight has been discussed in previous papers (Noci \& Maccari 1999; Ventura \& Spadaro 1999). The usual formula $v=c \Delta \lambda / \lambda$ rigorously applies only to the collisional component of a line, while, when applied to the radiative component, can lead to underestimate the inferred velocities for emission far from the plane of the sky. For this reason, the l.o.s. velocities here determined for lines which are a mixture of the radiative and collisional components (not directly disentangled), have to be considered as lower limits.

The estimated uncertainty in the line center positions is within 0.2 spectral pixels, which, for the considered lines, corresponds to a velocity of $\sim 5 \mathrm{~km} \mathrm{~s}^{-1}$. It was computed as the standard deviation of the line center positions for a set of 15 exposures collected during the mini-synoptic program of November 2, and relevant to the same heliocentric distance and polar angle in the unperturbed quiet corona. Note that we expect a higher uncertainty in velocity $\left(\simeq 10 \mathrm{~km} \mathrm{~s}^{-1}\right)$ at $2.2 R_{\odot}$ and above, owing to the double spectral binning adopted for these heliocentric distances (see Sect. 2).

The l.o.s. velocities determined by the O VI $1032 \AA$ and $\operatorname{Ly} \beta$ lines for the same bright features previously discussed in terms of the line intensity are reported in Figs. 11 and 12 , respectively.

The zero velocity level here adopted corresponds to the average l.o.s. velocity deduced for the background quiet coronal emission during the mini-synoptic program.

As for many other CMEs observed by UVCS, different features exhibit different Doppler shifts (e.g., Ciaravella et al. 2000). Moreover, the Doppler shift and the corresponding l.o.s. velocity of each feature changes with time: in some cases, for instance at $1.6 R_{\odot}$, the acceleration of a bright feature is associated with the deceleration of another one. All this is clearly evident for both the examined lines, whose Doppler shifts reveal a very complex velocity structure in the expanding material. The l.o.s. velocities relevant to different bright features may differ by several tens of $\mathrm{km} \mathrm{s}^{-1}$ in both the considered spectral lines, even if, above $2 R_{\odot}$, the differences appear 

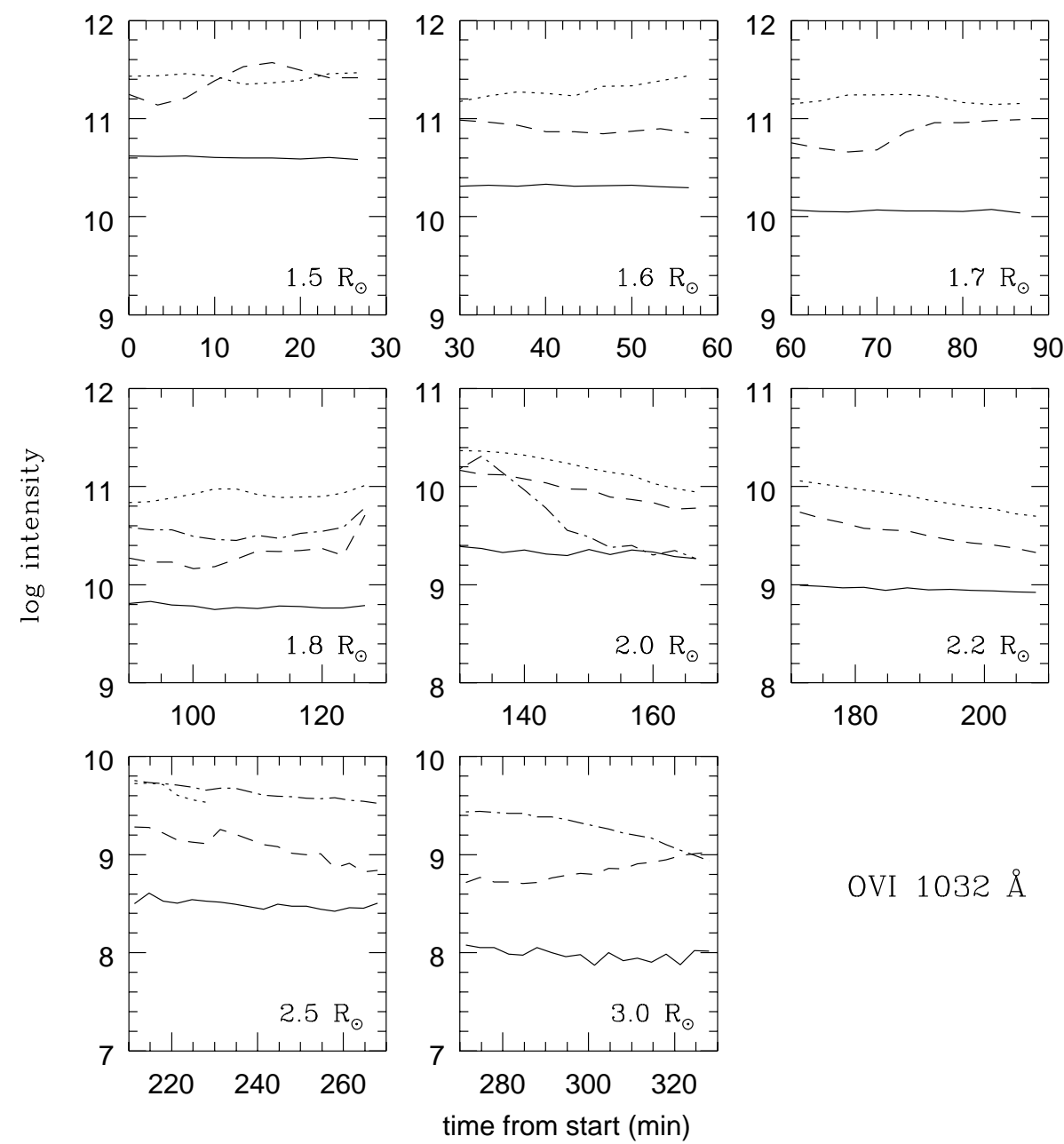

OVI $1032 \AA$

Fig. 9. O VI $1032 \AA$ line intensities (in photons $\mathrm{cm}^{-2} \mathrm{~s}^{-1} \mathrm{sr}^{-1}$ ) at each heliocentric positions of the radial scan of November 2 , 2000, as a function of the time. The different curves refer to different bright spots singled out along the UVCS entrance slit and identified on the basis of their distance from the slit center $\left(\mathrm{PA}=270^{\circ}\right)$ : \#1 at 4 arcmin North (short-dashed line), \#3 at 7 arcmin North (dotted line) and \#6 at 10 arcmin North (dotted-short-dashed line). All the spots are located at the top of the slit, i.e. toward increasing polar angles (the North Pole is identified by a PA equal to 360 degrees). The reference intensity level for the quiet background corona is also reported in each panel as a solid line.

significantly reduced, generally below $10-20 \mathrm{~km} \mathrm{~s}^{-1}$, and all the structures tend to exhibit similar velocities.

On the other side, the l.o.s. velocity deduced by the O VI line for a given feature identified along the slit is usually consistent with that deduced by the Ly $\beta$ line, with only a few exceptions found in limited time intervals at $1.6 R_{\odot}$ and $1.8 R_{\odot}$. These exceptions could be due to larger uncertainties in the line center determination caused by the poorer photon statistics characterizing the observations of the structures identified in the $\operatorname{Ly} \beta$ line.

It is worth noting, moreover, that we do not notice any evident correlation between the brightness of the structure and the corresponding l.o.s. velocity.

All the features exhibit positive Doppler shifts with respect to the coronal background wavelength reference and, although there are significant variations with time, particularly at $1.8 R_{\odot}$ and $2.0 R_{\odot}$ for structures identified in the part of the slit corresponding to larger polar angles, we do not note any change in the sign of the Doppler shifts. The l.o.s. velocities are usually above $100 \mathrm{~km} \mathrm{~s}^{-1}$, even if a few exceptions occur for some bright features observed at $1.7 R_{\odot}$ (in Ly $\beta$ only), $1.8 R_{\odot}$ and $2.0 R_{\odot}$ (only O VI data are available at this height). Note that these features are characterized by the large variations of Doppler shifts with time quoted above. At $2.2 R_{\odot}$ and higher, the l.o.s. velocities deduced by the Doppler shifts of the O VI line are between 100 and $150 \mathrm{~km} \mathrm{~s}^{-1}$ for all the structures singled out. The considerable differences (at least $50 \mathrm{~km} \mathrm{~s}^{-1}$ ) with the values obtained at $2.0 R_{\odot}$ are interesting. They could be due to the acceleration of the outflowing CME plasma and/or to its trajectory, which tends more and more to recede from the observer, so increasing the component of the flow velocity along the line of sight. A possible alternative explanation is that at $2.0 R_{\odot}$ the UVCS is observing the tail of the expanding CME material, characterized by lower velocity.

The position of the considered features at every single selected heliocentric distance does not vary perceptibly along the UVCS slit as a function of time, except at $3 R_{\odot}$, as we discuss later on. 


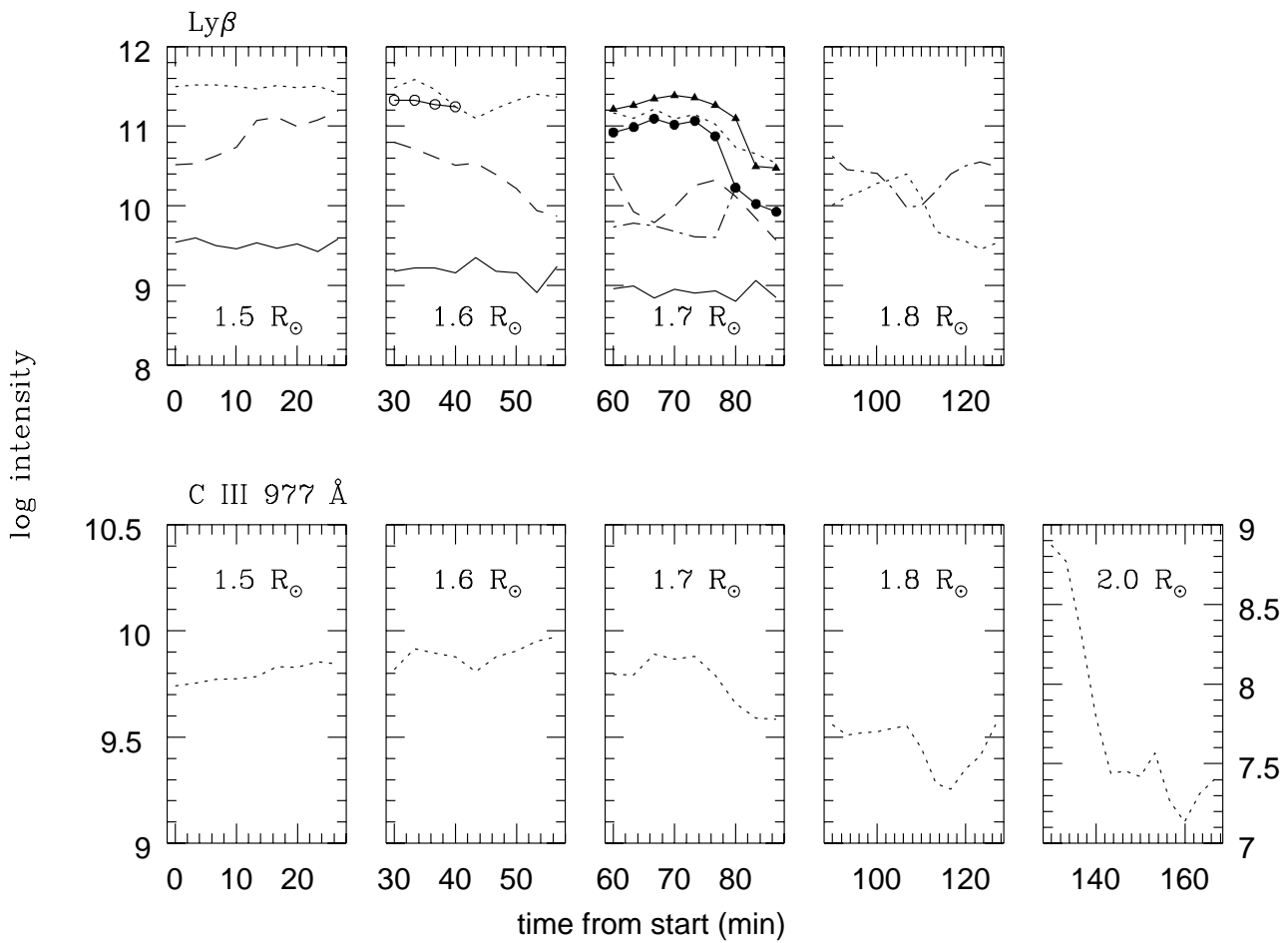

Fig. 10. As in Fig. 9, but for the Ly $\beta$ (top panels) and C III $977 \AA$ (bottom panels) lines. The Ly $\beta$ bright spots identified along the UVCS slit during the radial scan are \#1, at 4 arcmin North (short-dashed line), \#2 at 6 arcmin North (open circles), \#3 at 7 arcmin North (dotted line), \#4 at 7.7 arcmin North (solid triangles), \#5 at 8.4 arcmin North (solid circles) and \#6 at 10 arcmin North (dotted-short-dashed line) from the slit center (PA $270^{\circ}$ ). The reference intensity level for the quiet corona is also reported as a solid line. At $1.8 R_{\odot}$ the quiet corona Ly $\beta$ line is embedded in the noise whose estimated value is $2.5 \times 10^{8}$ photons $\mathrm{cm}^{-2} \mathrm{~s}^{-1} \mathrm{sr}^{-1}$. The C III intensity refers to a single bright spot located 7 arcmin North from the slit center (dotted line). In the quiet corona the C III line is undetectable at all heliocentric distances so that a reference level cannot be determined. An upper limit of the unperturbed corona C III emission can be fixed by the noise levels (in photons cm $\mathrm{ph}^{-2} \mathrm{sr}^{-1}$ ) i.e., $2.18 \times 10^{7}$ at $1.5 R_{\odot}, 5.30 \times 10^{7}$ at $1.6 R_{\odot}, 2.44 \times 10^{7}$ at $1.7 R_{\odot}, 9.24 \times 10^{6}$ at $1.8 R_{\odot}$ and $3.69 \times 10^{6}$ at $2.0 R_{\odot}$.

Comparing the positions along the slit of the bright features as they appear at different heights (even if in practice it is very difficult to identify specific features at the different heliocentric distances with confidence (cf. Ciaravella et al. 2000)), the CME material observed by UVCS appears to outflow along magnetic structures slightly curved $(<10 \mathrm{deg})$ to the North-West (see also the O VI $1032 \AA$ image reported in Fig. 3).

The various exposures collected at $3 R_{\odot}$, on the other side, show that the peaks of brightness shift along the slit towards its center by about 3.5 arcmin in less than one hour, hence with an apparent transverse velocity on the plane of the sky of about $45 \mathrm{~km} \mathrm{~s}^{-1}$. This shift is not associated to significant variations of the l.o.s velocity relevant to the considered structures, as shown in Fig. 11, and can be interpreted as the transit of an arch-like structure bent to North-West and to the opposite direction with respect to the observer, across the UVCS instantaneous field of view.

General information on the radial outflow velocity characterizing the event can also come from the Doppler dimming of the O VI resonance doublet: as discussed by Noci et al. (1987) and Dodero et al. (1998), the $1037 \AA / 1032 \AA$ intensity ratio is a powerful diagnostic tool for the speed of the expanding plasma. The values of this ratio obtained for the several bright knots identified along the slit at any heliocentric distance are generally equal to about 0.4 up to $1.8 R_{\odot}$, decreasing to about 0.3 at higher heights. These values indicate that the radial component of the outflow velocity is in both cases lower than $100 \mathrm{kms}^{-1}$ (see, e.g. Noci et al. 1987; Dodero et al. 1998). This component is significantly lower than the velocity of the CME leading edge deduced from LASCO observations (see Fig. 1). It is worth noting, however, that the UVCS observations cover only the footpoint segment, near the coronal base, of the large opening loop structure well visible in the images obtained by LASCO (see Fig. 2). Hence the flow of material there can be considerably slower than the expansion of the loop top at larger heliocentric distances.

\subsection{The CME of November 3}

\subsubsection{Spectra and line intensities}

Figure 13 reports a sequence of spectra taken on November 3 in the same two spectral regions and with the same spectral binning as for those reported in Fig. 8.

The first spectrum of the sequence was taken at $1.5 R_{\odot}$ (Figs. 13a, b). It does not show clear evidence of low- and 

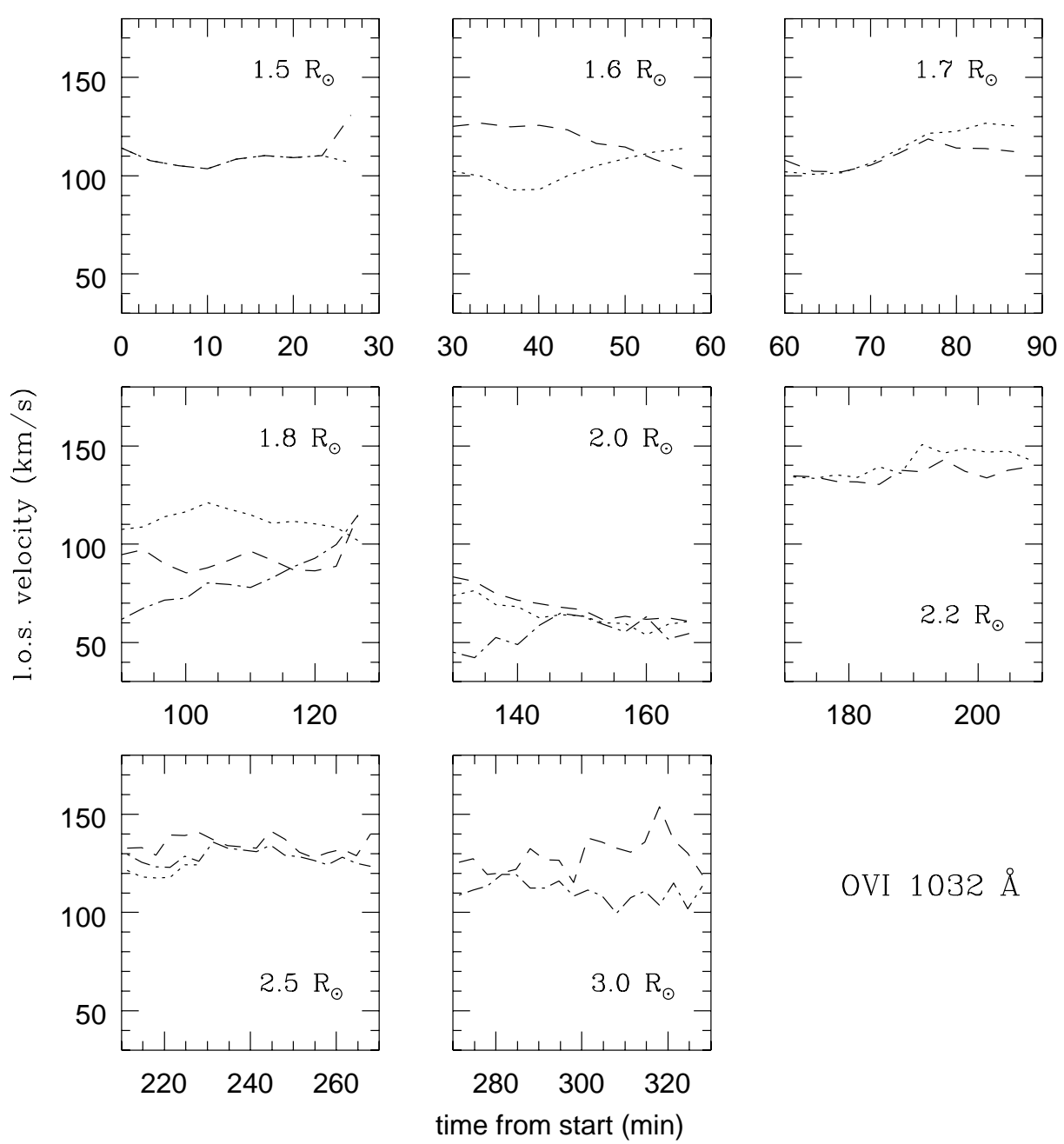

Fig. 11. Line of sight velocities derived from the O VI $1032 \AA$ Doppler shift, at each heliocentric distance of the radial scan of November 2, 2000, as a function of time. Positive velocities correspond to plasma receding from the observer. The different curves refer to the same bright spots identified along the slit as in Fig. 9.

intermediate temperature lines such as the H I Ly $\beta$ and Ly $\gamma$ lines and the C III line. Thus it is relative to the ambient corona. The front of the prominence eruption reaches the UVCS instantaneous field of view centered at $1.55 R_{\odot}$ at 19:56 UT, as it is evident from the appearance of the $\mathrm{Ly} \beta, \mathrm{Ly} \gamma$ and $\mathrm{C}$ III lines in the two spectral regions observed (Figs. 13c,d). The Ly $\beta$ and C III intensities are almost comparable to those of the O VI resonance doublet, whose peak intensity ratio O VI 1037/O VI $1032 \AA$ has clearly increased, reaching the value of 0.5 , in comparison with the unperturbed value of 0.37 (Fig. 13a). At $1.6 R_{\odot}(21: 13 \mathrm{UT})$ (Figs. 13e,f) the Ly $\beta$ becomes 20 times brighter than at $1.55 R_{\odot}$, but it is still fainter than the $\mathrm{O}$ VI $1032 \AA$ line. The C III is the most intense line in the spectrum, being even brighter than the coronal OVI resonance doublet. This fact suggests that a massive prominence eruption ejected cool plasma of obvious chromospheric origin into the coronal ambient. At 21:17 UT the UVCS moves back to $1.5 R_{\odot}$ and starts again its radial scan up to $1.6 R_{\odot}$. At 22:03 UT (Figs. 13g, h) the CME faded out and the spectra are again typical of the ambient corona.
Figure 14 shows a series of O VI $1032 \AA$ long -slit profiles observed on November 3. The first image shows the unperturbed corona. A small region, one order of magnitude brighter than the unperturbed corona, appears in the second image when the erupting prominence enters the instantaneous field of view of UVCS together with some other fainter spots. They progressively intensify with time, as the expanding prominence crosses the UVCS slit. Some new bright knots develop, which irregularly appear and disappear in different locations along the slit as new material reaches $1.6 R_{\odot}$. At 21:59 UT the bright regions begin to fade and the long-slit profile become very similar to that typical of the unperturbed corona.

The intensity of the observed lines during the evolution of the prominence eruption is reported in Figs. 15 and 16.

Since UVCS was able to follow the development of the CME from its beginning until the end, the intensity values referring to the unperturbed corona were computed in two ways: a) integrating over the full length of the slit, before and after the CME crossing through the instantaneous field of view of the instrument; b) considering only a less bright section of the slit, far away the bulk of the CME 


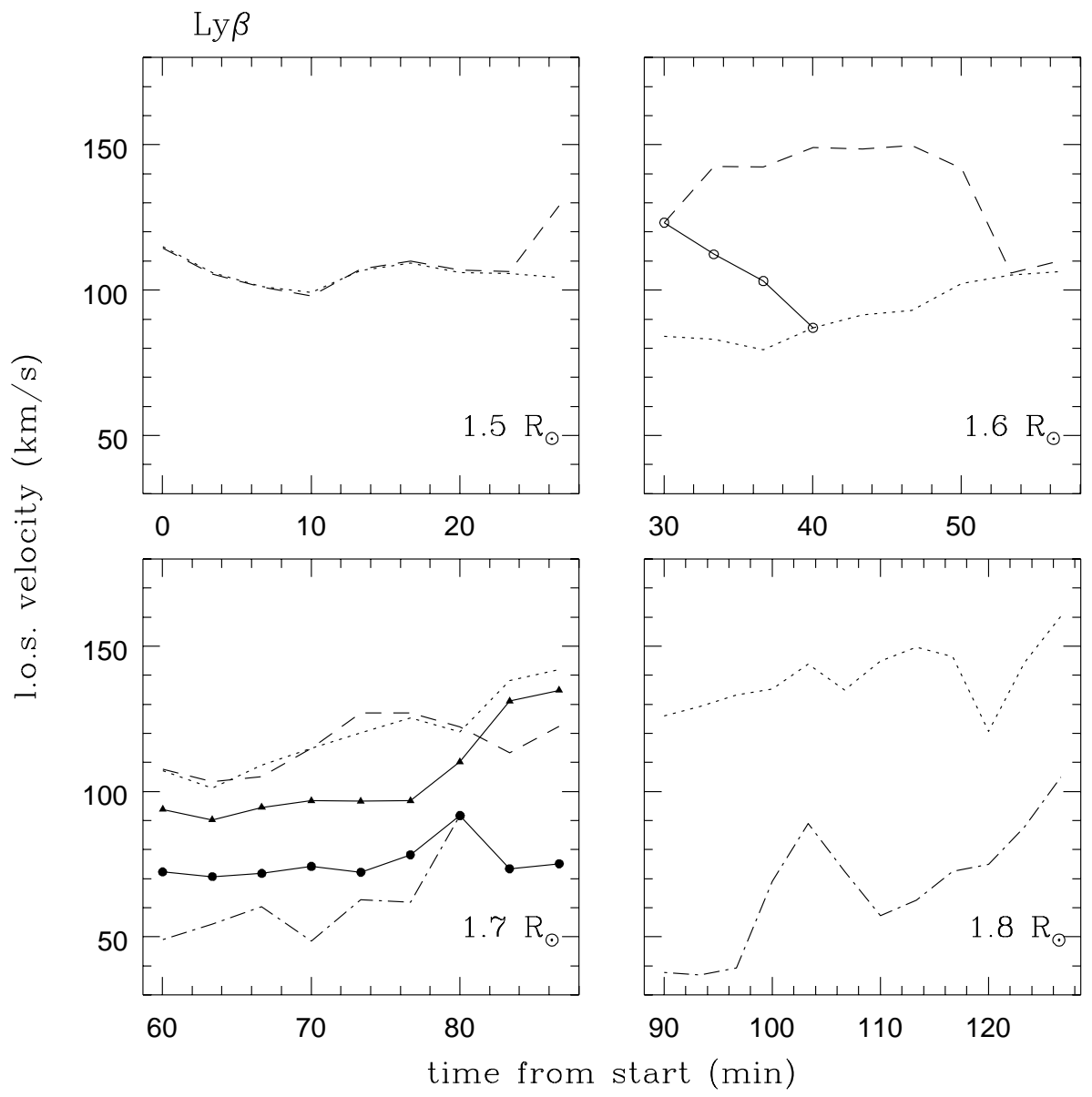

Fig. 12. As in Fig. 11, but for the Ly $\beta$ line. Different curves refer to the same bright spots identified along the slit as in Fig. 10.

brightening, during the evolution of the event, as in the case of the CME of November 2 .

The O VI $1032 \AA$ intensity at $1.5 R_{\odot}$ is fairly constant, since the CME front has not yet reached the UVCS instantenous field of view. The effect of the CME transit is evident at $1.55 R_{\odot}$. As for the event of November 2 , several spot-like bright features appear along the slit (see Fig. 14); hence we consider the behaviour of the relevant intensities in detail. We note that the intensity in some bright knots suddenly increases by up to one order of magnitude in the last exposure (spots \#3 and \#4). At $1.6 R_{\odot}$ the intensity distribution along the slit is more complex, with the development of many bright knots caused by the CME expansion. Some of them evolve more rapidly than the others and the brightest ones reach intensity values more than one order of magnitude higher than in the quiet corona (spots \#2 and \#3). When the UVCS restarts its radial scan at $1.5 R_{\odot}$, the bulk of the event appears to have left the field of view of the instrument. Some localized brightenings still persist (\#3 and \#5), but their intensity values decrease progressively towards the typical values for the quiet corona emission. At $1.55 R_{\odot}$ and $1.6 R_{\odot}$ the O VI $1032 \AA$ emission is that previously measured in the unperturbed corona.

The Ly $\beta$ and C III intensities are reported in Fig. 16 and refer only to those heliocentric distances where the two lines were detectable. At $1.55 R_{\odot}$ the behaviour of the intensity distributions of both lines shows similar characteristics determined by the entry of the CME in the UVCS instantaneous field of view: in the two bright spots identified along the slit (\#3 and \#4), the Ly $\beta$ intensity rapidly increases by more than two orders of magnitude, while the C III $977 \AA$ intensity increases in the only bright spot identified (\#4) by about a factor of forty over the time interval of the observations. At $1.6 R_{\odot}$ the $\mathrm{Ly} \beta$ intensity distribution along the slit become more complex with the presence of four distinct brightenings, which irregularly evolve in a way very different from each other. The C III intensity, conversely, shows a simple behaviour. The spot \#4 brightens up to one order of magnitude in six exposures, then remains approximately constant until the end of the first radial scan. When the UVCS restarts its radial scan at $1.5 R_{\odot}$, the $\mathrm{Ly} \beta$ intensity appears nearly constant in both the bright knots (\#3 and \#5) still surviving, while the C III continues to weaken becoming an order of magnitude fainter in the last exposure.

\subsubsection{Velocity structure}

Figure 17 reports the l.o.s. velocities determined by the O VI $1032 \AA$ (top panels) and Ly $\beta$ (bottom panels) lines for the bright features identified along the spectrometer slit during the CME of November 3. The zero reference level is deduced from the mini-synoptic observations of 

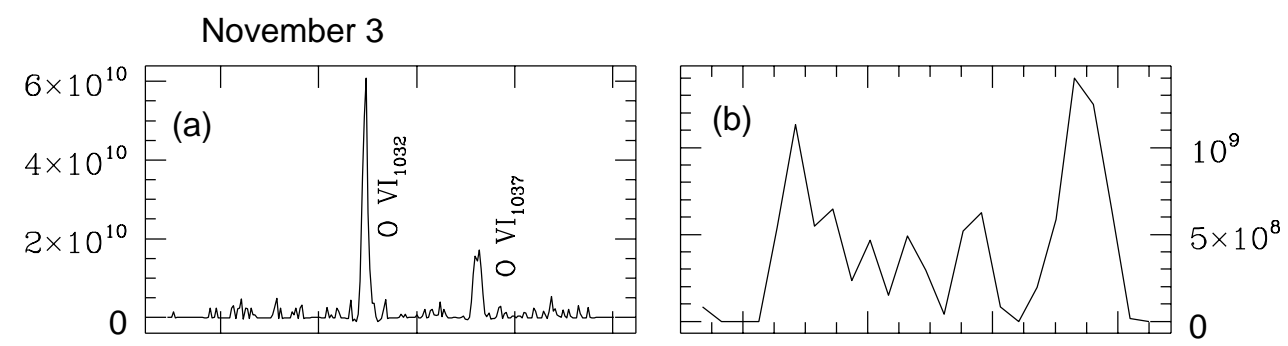

त)
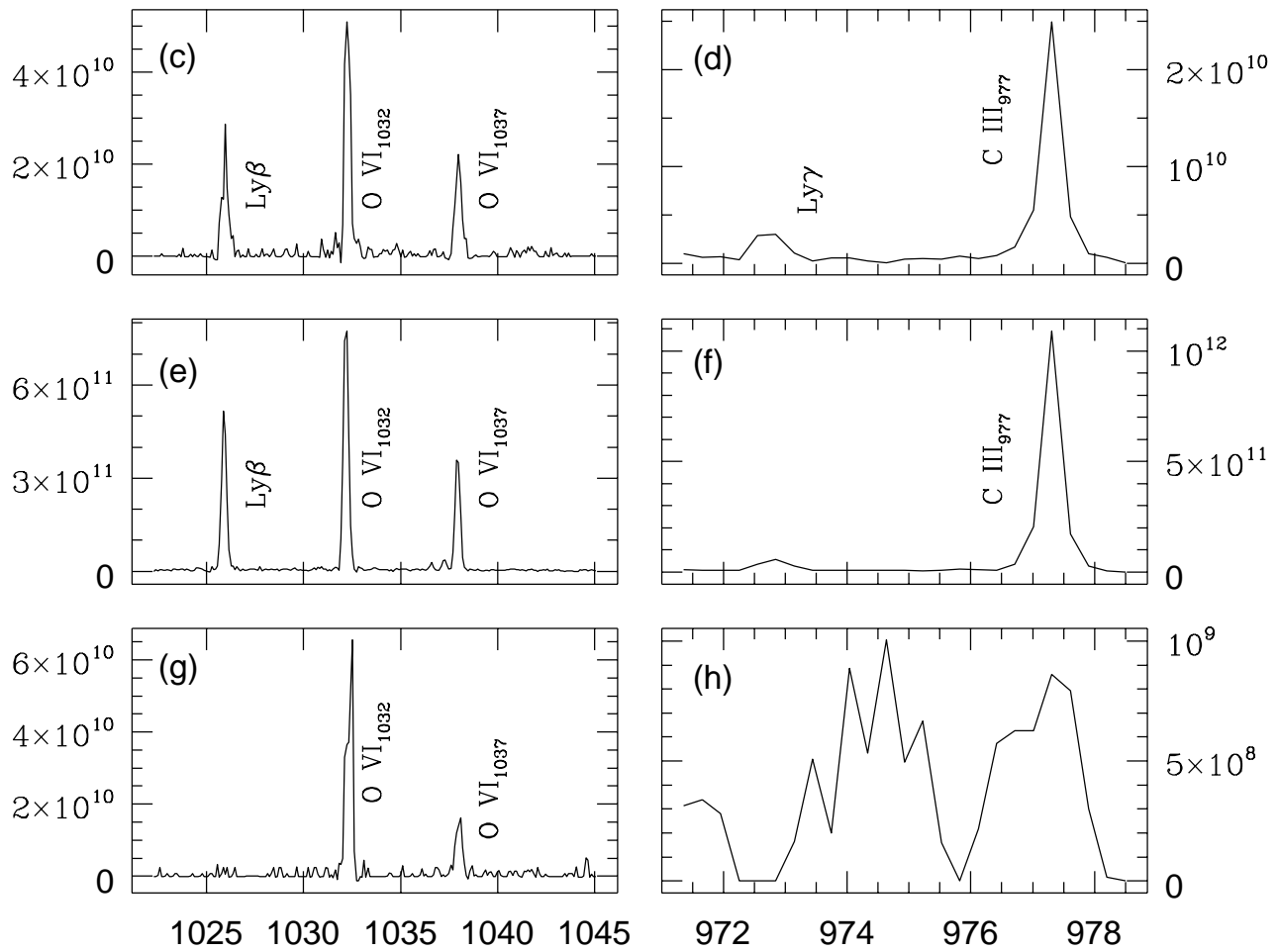

$\lambda(\AA)$

Fig. 13. Sequence of spectra extracted from the UVCS data of November 3, 2000 in the wavelength regions $1022-1045 \AA$ and 970-978 $\AA$. The panels refer to the following heliocentric distances and initial exposure times, respectively: a,b) $1.5 R_{\odot}$, 18:17 UT, c,d) $\left.1.55 R_{\odot}, 19: 56 \mathrm{UT}, \mathbf{e , f}\right) 1.6 R_{\odot}, 21: 13 \mathrm{UT}$ and $\left.\mathbf{g}, \mathbf{h}\right) 1.5 R_{\odot}, 22: 03 \mathrm{UT}$ (second scan). Note the changes in the vertical scale among different panels. All the spectra have been obtained integrating along the slit over the spatial extent $\left(42^{\prime \prime}\right)$ of the very bright spot \#3, located at 1.4 arcmin South from the slit center (see Fig. 14).

the day, as in the case of the event of November 2, and does not differ significantly from the value used for the first CME.

Since UVCS caught the beginning of the transit of the CME expanding material across its instantaneous field of view, we note the sharp change of the l.o.s. velocity relevant to some features with respect to the positive l.o.s. velocity characterizing the unperturbed corona above the South Pole $\left(\simeq 60 \mathrm{~km} \mathrm{~s}^{-1}\right)$ : at $1.55 R_{\odot}$ two bright O VI features (\#3 and \#4) appear near the center of the spectrometer slit approximately $90 \mathrm{~min}$ after the start of the radial scan, and reduce their velocity to nearly $20 \mathrm{~km} \mathrm{~s}^{-1}$ in about $10 \mathrm{~min}$. Also in the $\operatorname{Ly} \beta$ line, at $1.55 R_{\odot}$, we can distinguish a feature brighter than the coronal background emission near the slit center (spot \#4), which already moves with a velocity of about $10 \mathrm{~km} \mathrm{~s}^{-1}$ when it first appears, 80 min after the start of the radial scan. It then splits up into two features (\#3 and \#4) whose l.o.s. velocities have opposite signs and differ by $\sim 20 \mathrm{~km} \mathrm{~s}^{-1}$.

At $1.6 R_{\odot}$, similarly, there is the development of quite a few bright features in the O VI line, each with a different temporal evolution, which progressively get velocities of only some tens of $\mathrm{km} \mathrm{s}^{-1}$, still receding from the observer, and then approach again the velocity characterizing the unperturbed corona above the South Pole. The $\operatorname{Ly} \beta$ velocity structure is more complicated: we note both motions receding from and towards the observer, even with speeds significantly different from those in O VI. Moreover the behaviour of the various structures is rather irregular, probably as a consequence of larger uncertainties in the Doppler shifts, due to the poorer photon statistics characterizing the observation of the structures identified in the Ly $\beta$ line.

When UVCS starts again to scan radially the corona above the South Pole, observing back at $1.5 R_{\odot}$, only the 


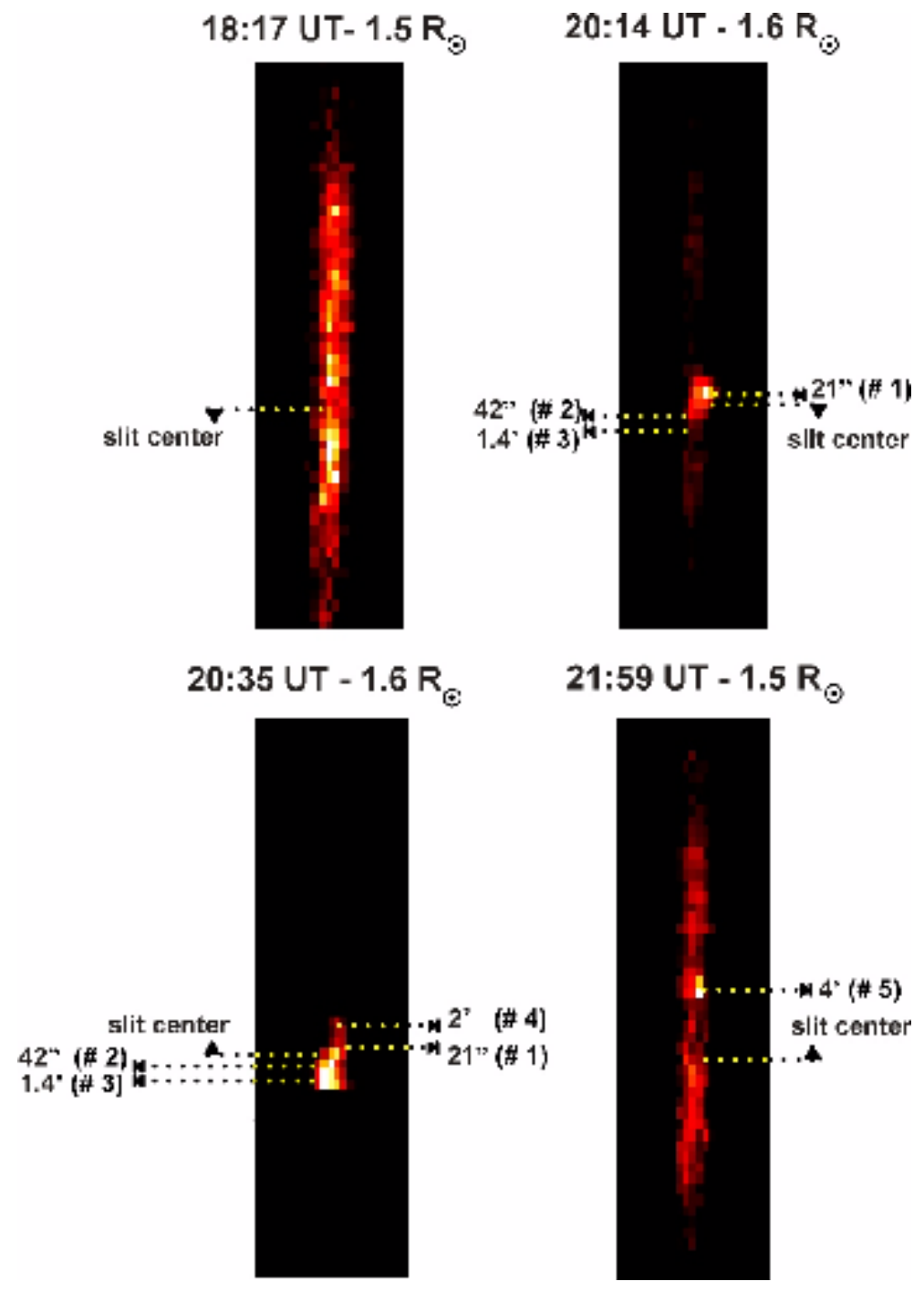

Fig. 14. UVCS long-slit O VI $1032 \AA$ profiles obtained on November 3, 2000 during the prominence eruption. Each image was scaled to its brighest pixel. The vertical and horizontal axes are the spatial coordinates along the slit and the wavelengths, respectively. Shorter wavelengths are at right and smaller polar angles at the top of each panel. The vertical extent is 40 arcmin, the covered wavelength range is $3.76 \AA$. The slit is centered at a PA equal to 200 degrees. The initial UT time of each exposure and the heliocentric distance are reported on the top of each panel. See Fig. 6 for the UVCS slit position at the time of observation in relation to the solar disc. The slit center together with the bright spots identified and numbered progressively on the basis of their distance from it are also shown.

CME tail is still in its field of view, and the features singled out in O VI (\#3 and \#5) move along the line of sight with velocities around $50 \mathrm{~km} \mathrm{~s}^{-1}$, reaching the initial l.o.s. velocity characterizing the corona before the transient event during the successive exposures. The subsequent exposures no longer show evidence of the CME transit. We notice a similar behaviour in Ly $\beta$, although the structures identified in this line exhibit a steeper change in the l.o.s. velocity.

Unless there is a systematic error in the evaluation of Doppler shifts, this result could imply the existence along the same line of sight of different, isolated CME structures, preferentially emitting in different spectral lines, hence characterized by differences in the plasma temperature and/or density, which move with different l.o.s. velocities.
While it is difficult to detect systematic shifts of the considered bright features along the UVCS slit during the first radial scan, owing to their complex and irregular evolution from one exposure to another, during the second scan, at $1.5 R_{\odot}$, the peaks of brightness appear to depart regularly from the slit center towards higher polar angles by about 2 arcmin in $2000 \mathrm{~s}$, hence with an apparent transverse velocity on the plane of the sky of about $40 \mathrm{~km} \mathrm{~s}^{-1}$. This shift can be interpreted as the transit across the UVCS instantaneous field of view of the arched plasma structure of the erupting prominence shown in Fig. 7, whose leg at higher polar angles appears brighter in the observed EUV lines.

The O VI intensity ratio computed for each bright knot identified along the slit during the temporal evolution of the prominence eruption, gives us some coarse indication 
OVI $1032 \AA$
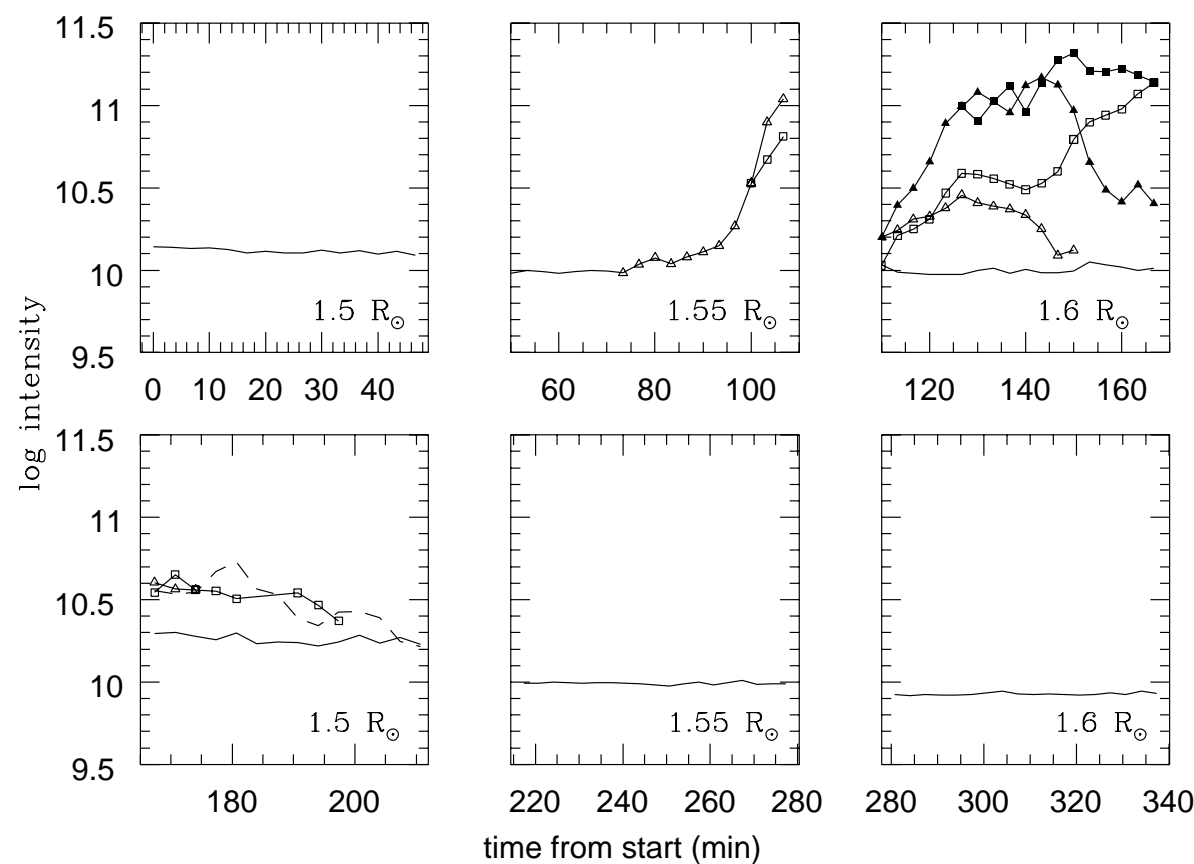

Fig. 15. As in Fig. 9, but for the event of November 3, 2000. The bright spots identified along the slit during the radial scan are located at: 21 arcsec North \#1 (solid triangles), 42 arcsec South \#2 (solid squares), 1.4 arcmin South \#3 (open squares), 2 arcmin North \#4 (open triangles) and 4 arcmin North (short-dashed line) \#5, respectively, from the slit center (see Fig. 14). The solid line refers to the emission of the unperturbed corona.

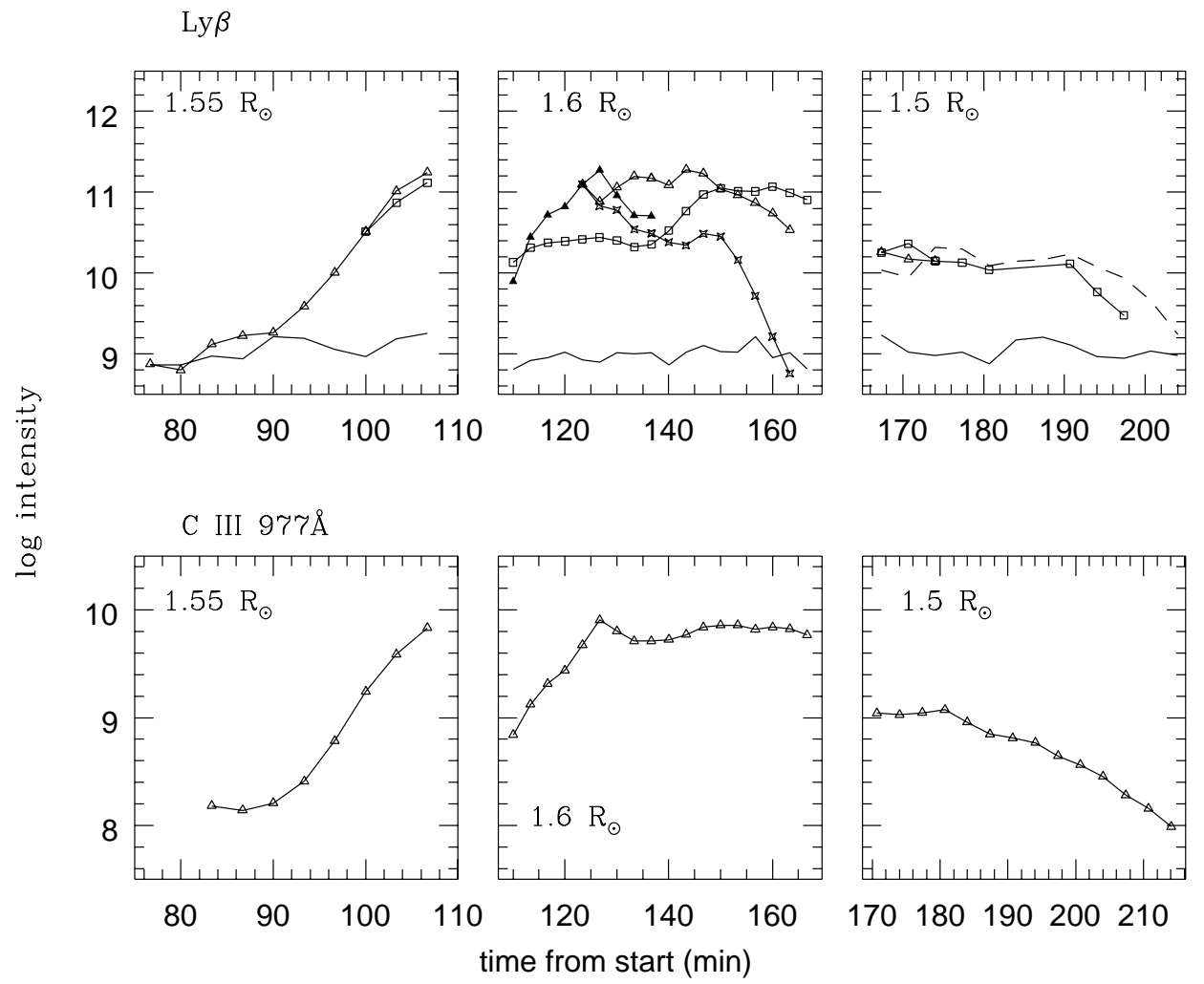

Fig. 16. As in Fig. 10, but for the Ly $\beta$ (top panels) and C III $977 \AA$ (bottom panels) lines. Different curves refer to the same bright spots identified along the slit as in Fig. 15. A bright spot (hereafter numbered as \#6) identified in the Ly $\beta$ radiation and not present in the O VI $1032 \AA$ emission is located at $42 \operatorname{arcsec}$ North (starred line) from the slit center. The noise level determined in the wavelength region of the C III line is $1.0 \times 10^{7}$ at all heliocentric distances considered. 
O VI $1032 \AA$

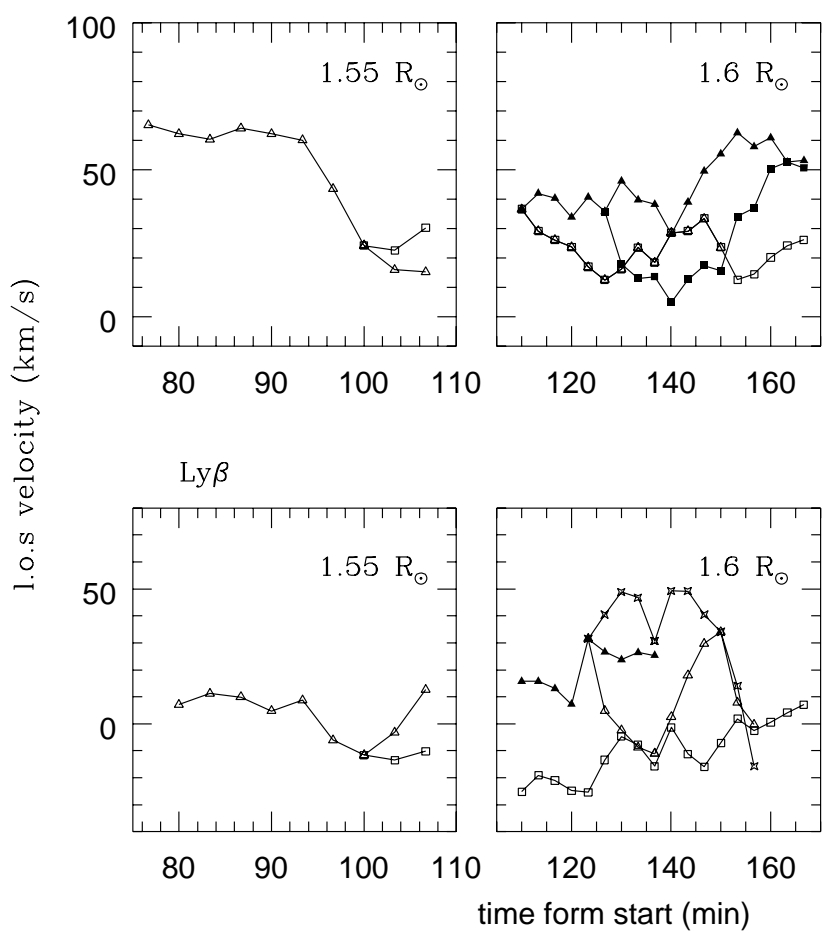

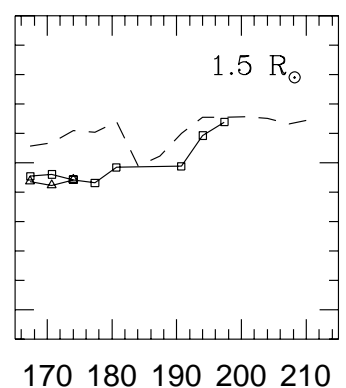

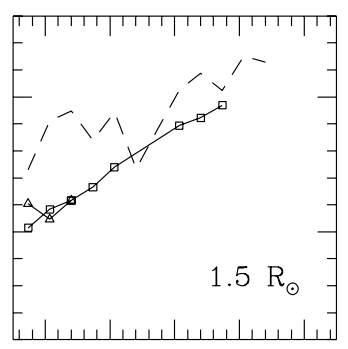

170180190200210

Fig. 17. Line of sight velocities derived from the O VI $1032 \AA$ (top panels ) and Ly $\beta$ (bottom panels) lines Doppler shifts, at different heliocentric distances and as a function of the time, referring to the prominence eruption of November 3, 2000. Positive velocities correspond to plasma receding from the observer. Symbols refer to the same bright spots as in Figs. 15 and 16.

on the radial outflow velocity characterizing the event. Before and after the CME crossed the UVCS field of view the value of this ratio is in the range $0.3-0.4$ for all the considered bright spots while during the event it increases to about 0.5 . This fact indicates that the radial component of the outflow velocity during the event reachs $\simeq 100 \mathrm{~km} \mathrm{~s}^{-1}$ (see Noci et al. 1987; Dodero et al. 1998). Hence we note an acceleration with respect to the phases of the prominence eruption observed by EIT (see Fig. 7 and the related discussion). A possible alternative explanation is that the denser CME material causes the collisional component dominate the emission of $\mathrm{O}$ VI lines, giving an intensity ratio equal to 0.5 (e.g., Noci et al. 1987).

\section{Conclusions}

We analysed UVCS/SOHO observations of two coronal mass ejections which occurred consecutively on November 2 and 3, 2000 (during the MEDOC campaign \#6), in order to get information about the distributions of some EUV line intensities and of flow velocities in the solar plasma ejected during these transient events. The former CME followed the eruption of a large filament structure in an active region close to the West limb, and was observed by UVCS after the transit of its leading edge across the field of view; the latter one was caused by the eruption of a prominence near the South Pole, and was observed while starting its expansion in the heliosphere.

The ejected material was very bright in the lowand intermediate-temperature lines for both the event, whereas no significant emission was detected in the high-temperature coronal lines observable in the selected wavelength regions (e.g., Si XII $521 \AA$ at 2nd order, [Fe XVIII] $974 \AA$ ). This indicates the presence of plasma in the range of temperature $10^{4.5}-10^{5.5} \mathrm{~K}$. EIT observations in the Fe line bands, on the other hand, show emission even at $\simeq 1.5 \times 10^{6} \mathrm{~K}$ for both CMEs, putting into evidence that plasma at different temperatures is ejected during the events.

The O VI and Ly $\beta$ intensity distributions relevant to the two studied cases reveal a complex, rapidly evolving, morphology of the CME, with the development of several bright strands at different positions, rather irregularly distributed inside the CME structure. Moreover, these features exhibit a brightness temporal evolution significantly different from each other.

The l.o.s. velocities determined for each bright element also have different values, spanning a wide range, as well as different temporal behaviours, which gives a complex picture of the plasma kinematics characterizing these coronal mass ejections.

It is possible, however, to determine some global properties marking the two considered events out. The CME of November 2 is an example of transient event where the plasma is generally receding from the observer at speeds up to $100-150 \mathrm{~km} \mathrm{~s}^{-1}$, while its velocity on the plane of the sky measured along the spectrometer slit direction is rather smaller $\left(\sim 40 \mathrm{~km} \mathrm{~s}^{-1}\right)$. The Doppler dimming of the $\mathrm{O}$ VI resonance doublet gives radial components of the outflow velocity below $100 \mathrm{~km} \mathrm{~s}^{-1}$ as well. Therefore, the structure observed by the UVCS could consist of a 
bundle of different filaments which looks like the leg, near the coronal base, of an opening large loop, departing from the observer and slightly curved to North-West, whose top is well visible at larger heliocentric distances in the images obtained by LASCO (Fig. 2) and expands outwards with velocities well above $100 \mathrm{~km} \mathrm{~s}^{-1}$.

As for the event of November 3, the ejecta seem to expand in the corona near the South Pole, where a steady outflow is already present with a l.o.s. velocity component of $\simeq 60 \mathrm{~km} \mathrm{~s}^{-1}$. They form a complex fan of rays above the erupting prominence, whose arch-shaped structure transits across the UVCS instanteneous field of view during the final phases of the CME observation.

The Doppler dimming of the O VI resonance doublet results in values for the radial component of the outflow velocity reaching about $100 \mathrm{~km} \mathrm{~s}^{-1}$, thus evidencing an acceleration of the ejected material with respect to the phases of the prominence eruption observed by EIT.

These, as well as other SOHO observations, point to the need for more detailed models of CMEs, in particular models that treat the thermal state of the plasma and that follow the field structure and plasma flow in a selfconsistent manner.

Acknowledgements. This work was made possible by the UVCS/SOHO instrument and Flight Operations team. The UVCS is the result of a collaborative effort between NASA and the Agenzia Spaziale Italiana (ASI), with a Swiss participation.

R. Ventura and D. Spadaro wish to thank J. C. Vial, Responsable of the Solar Team and K. Bocchialini, Scientific Director of the Institute d'Astrophysique Spatiale in Orsay Paris, and all the staff of the MEDOC center for kind hospitality. They also thank J. P. Delaboudiniere and S. Plunkett for kindly providing important information on EIT and LASCO CME observations of November 2 and 3, 2000.

This work was in part supported by the Agenzia Spaziale Italiana.

\section{References}

Antiochos, S. K., Devore, C. R., \& Klimchuk, J. A. 1999, ApJ, 510,485
Antonucci, E., Kohl, J. L., Noci, G., et al. 1997, ApJ, 490, L186

Brueckner, G. E., Howard, R. A., Koomen, M. J., et al. 1995, Sol. Phys., 162, 357

Chen, J. 1996, JGR A, 101, 27499

Ciaravella, A., Raymond, J. C., Thompson, B. J., et al. 2000, ApJ, 529, 575

Delaboudiniere, J.-P., Artzner, G. E., Brunaud, J., et al. 1995, Sol. Phys., 162, 291

Dodero, M. A., Antonucci, E., \& Martin, R. 1998, Mem. SAIt, 69, 757

Domingo, V., Fleck, B., \& Poland, A. 1996, Sol. Phys., 162, 1

Durasova, M. S., Fridman, V. M., \& Sheiner, O. A. 1999, ESA SP, 448, 2, 979

Gardner, L. D., Kohl, J. L., Daigneau, P. S., et al. 1996, Proc SPIE, 2831, 2

Hundhausen, A. J. 1988, Proceedings of the Sixth International Solar Wind Conference, ed. V. J. Pizzo, T. E. Holzer, \& D. G. Sime (HAO/NCAR), 1, 181

Hundhausen, A. J. 1997, in Cosmic Wind and the Heliosphere, ed. J. R. Jokipii, C. P. Sonett, \& M. S. Giampapa (Tucson: Univ. Arizona Press), 259

Kohl, J. L., Esser, R., Gardner, L. D., et al. 1995, Sol. Phys., 162,313

Kohl, J. L., Gardner, L. D., Fineschi, S., et al. 1997a, Adv. Space Res., 20, 3

Kohl, J. L., Noci, G., Antonucci, E., et al. 1997b, Sol. Phys., 175,613

Low, B. C. 1994, Phys. Plasmas, 1, 1684

Low, B. C. 1996, Sol. Phys., 167, 271

Maia, D., Vourlidas, A., Pick, M., et al. 1999, JGR, 104 (A6), 12507

Maia, D., Pick, M., Vourlidas, A., \& Howard, R. 2000, ApJ, 528, L49

Moore, R. L., \& Roumeliotis, G. 1992, in Eruptive Solar Flares, ed. Z. Svestka, B. V. Jackson, \& J. Feynmann (Washington: Am Geophys. Union), 119

Noci, G., \& Maccari, L. 1999, A\&A, 341, 275

Noci, G., Kohl, J. L., \& Withbroe, G. L. 1987, ApJ, 315,706

Panasyuk, A. V., et al. 2001, in preparation

Romoli, M., et al. 2001, in preparation

Sturrock, P. A. 1989, Sol. Phys., 121, 387

Ventura, R., \& Spadaro, D. 1999, A\&A, 341, 264

Wagner, W. J. 1984, ARA\&A, 22, 267 